\title{
Self-Sustained Clusters as Drivers of Computational Hardness in p-spin Models
}

\author{
Jacopo Rocchi* and David Saad ${ }^{\dagger}$ \\ Nonlinearity and Complexity Research Group, Aston University, Birmingham B4 7ET, United Kingdom \\ Chi Ho Yeung $\ddagger$ \\ Department of Science and Environmental Studies, \\ The Education University of Hong Kong, Hong Kong
}

(Dated: May 15, 2017)

\begin{abstract}
While macroscopic properties of spin glasses have been thoroughly investigated, their manifestation in the corresponding microscopic configurations is much less understood. Cases where both descriptions have been provided, such as constraint satisfaction problems, are limited to their ground state properties. To identify the emerging microscopic structures with macroscopic phases at different temperatures, we study the $p$-spin model with $p=3$. We investigate the properties of selfsustained clusters, defined as variable sets where in-cluster induced fields dominate over the fields induced by out-cluster spins, giving rise to stable configurations with respect to fluctuations. We compute the entropy of self-sustained clusters as a function of temperature and their sizes. In-cluster and out-cluster field properties support the observation of slow-evolving spins in spin models. These findings are corroborated by numerical studies in finite-size systems at low temperatures.
\end{abstract}

Spin glass models of disordered systems are characterized by a rich structure of the free-energy landscape and slow dynamics at low temperature. Mean field analyses $[1,2]$ typically provide a characterization of the state of the system based on a set of macroscopic order parameters and have provided many interesting and counterintuitive insights $[3,4]$. Symmetry properties of the resulting order parameters lead to distinct classes of systems termed One-step Replica Symmetric Breaking (1RSB) $[5,6]$ and Full Replica Symmetry Breaking [3, 7] models; the symmetries reflect the organization of states in the free-energy landscape and correspond to an increasingly more complex structure.

Phase transitions in spin-glass systems have been extensively studied within the macroscopic system representation. In particular, models with 1-RSB are common in physics, for instance in structural glass forming liquids [8-11], as well as in a range of hard-computational problems in computer science, such as Constraint Satisfaction Problems (CSP) [3, 12, 13]. They typically undergo a sequence of structural transitions when the temperature is decreased: while at temperatures above the dynamical transition $T>T_{d}$ the system is dominated by a paramagnetic (liquid) state; at lower temperatures $T<T_{d}$ an exponential number (in the number of variables) of TAP (Thouless-Andersson-Palmer) states emerge [14, 15], leading to a transition beyond which ergodicity breaks. This dynamical glass transition is characterized by a non-decaying spin-spin correlation function in disagreement with the static equilibrium zero value [16-20]. As the temperature decreases further the number of such states, whose logarithm is called complexity, decreases. Eventually, the complexity vanishes

\footnotetext{
* j.rocchi@aston.ac.uk

† d.saad@aston.ac.uk

¥ chyeung@eduhk.hk
}

at $T_{K}$, termed the Kauzmann transition in the physics of glass forming liquids and signals a true second-order phase-transition.

While the different temperature regimes are well understood in terms of the (free-) energy landscape, it is much more difficult to describe the manifestation of such changes in microscopic configurations. Interesting cases where this connection is clearer are CSP, whose solutions are organized in disconnected groups which contain frozen variables in intermediate regimes before the satisfiability transition [21-26]. Frozen variables take the same value in all the solutions of individual groups.

Since CSP are often studied in the context of hard optimization problems at $T=0$, the main external parameter considered is the ratio of constraints to variables $\alpha$ rather than temperature [22]. In general, frozen variables appear for higher $\alpha>\alpha_{d}$ values [23] with the exception of particular cases such as $k$-XORSAT, $k>2$, where they appear at the dynamical transition $\alpha_{d}[23,25]$. Nevertheless, this understanding is limited to optimal solutions, i.e. ground states of CSP, where as their manifestation at non-zero temperatures remains unclear.

In this work we investigate the existence of frozenlike variables in finite-temperature systems. More precisely, we look for clusters of spin variables that exhibit slow dynamics; the mere existence of such clusters is not guaranteed a priori. Somewhat similar problems have been studied in the context of spin glasses on random graphs $[27,28]$ and in finite dimensional lattices $[29,30]$ showing that it is possible to interpret non-equilibrium dynamical properties in terms of structural properties of the ground states of these systems. These works rely on the notion of rigidity lattice [31] and the corresponding analyses can be usually done in small systems. Our approach, while aiming at similar goals, relies on a different concept and can be used to analyze spin models via mean field methods. The central objects of our approach are Self-Sustained Clusters (SSC), introduced in the study 
of the SK model [32]. Pictorially, SSC can be considered as stable components of the system that make relaxation prohibitively slow at low temperature.

Our analysis is carried out within the framework of fully connected Ising $p$-spin model [33] with $p=3$, whose $T=0$ limit coincides with the $k$-XORSAT problems with $k=3$. To be precise, $k$-XORSAT is usually studied on sparse topologies but nevertheless the qualitative behaviour of the model does not change in densely connected systems. While this model belongs to the 1-RSB class, it can be studied in a non-trivial phase by using a simple ansatz for the order parameters, which nevertheless exhibits interesting and non-trivial dynamical properties. We compute the entropy of these clusters as a function of their size, and characterize their properties for different temperatures. Additionally, we study their stability by computing the distribution of the local field in typical SSC and show that SSC found at low temperatures can be considered as clusters of slow-evolving spins.

Model: The Hamiltonian of the fully connected 3-spin model $[16,17,21,33]$ with Ising variables is given by

$$
H=-\sum_{i<j<k=1}^{N} J_{i j k} s_{i} s_{j} s_{k},
$$

where $J_{i j k}$ are i.i.d. Gaussian random variables, with mean 0 and variance $p ! /\left(2 N^{p-1}\right)$, where $N$ is the number of spins. A brief description of the phase transitions of the model is provided in the Supplemental Material (SM) as well as in $[13,34,35]$. Be $\underline{s}$ an arbitrary spin configuration and let us use $\sigma$ variables to define the cluster membership per spin. Given a configuration $\underline{s}$ and cluster $C$, we assign $\sigma=+1$ for in-cluster spins and $\sigma=-1$ for out-cluster spins. To define the notion of a SSC, we write the local field $h_{i}$ acting on spin $s_{i}$ in a 3 -spin model as the sum of three contributions,

$$
h_{i}=\frac{1}{2} \sum_{j, k} J_{i j k} s_{j} s_{k}=\frac{1}{2}\left(u_{i}+v_{i}+w_{i}\right),
$$

$$
\begin{aligned}
& \text { where, } u_{i}=\sum_{j \in C, k \in C} J_{i j k} s_{j} s_{k}, \quad v_{i}=\sum_{j \notin C, k \notin C} J_{i j k} s_{j} s_{k} \\
& \text { and } w_{i} / 2=\sum_{j \in C, k \notin C} J_{i j k} s_{j} s_{k} .
\end{aligned}
$$

Similar definitions of the fields for general $p$-spin model are found in the SM. These three field contributions correspond to fields induced from within the cluster (in-in contribution, $u_{i}$ ), from outside the cluster (out-out contribution, $v_{i}$ ) and by both in- and out-cluster spins (inout contribution, $w_{i}$ ). A SSC is a group of spins such that, for each spin, the in-in contribution dominates the field $h_{i}$ with respect to all other contributions, and the following condition is satisfied

$$
u_{i}^{2}>\left(v_{i}+w_{i}\right)^{2}+\epsilon \quad \forall i \in C,
$$

where the positive external parameter $\epsilon$ can be arbitrarily adjusted to probe the SSC of specific strength. Selfsustained clusters are of interest since for $i \in C$, local fluctuations, giving $O(1 / N)$ contributions, do not change the relative importance of $u_{i}^{2}$ and $\left(v_{i}+w_{i}\right)^{2}$ and, thus, do not change the direction of the corresponding local fields. These clusters are therefore more stable compared to random groups of spins and offer a different perspective on the dynamical slowing down observed at low temperatures.

To count the number of SSC of size $r N$ in a given configuration $\underline{s}$ we define the entropic function of $r$

$\mathcal{S}\left(r \mid \underline{s},\left\{J_{i j k}\right\}\right)=N^{-1} \log \sum_{\underline{\sigma}} \mathbb{I}_{\underline{\sigma}}(\underline{s}) \delta\left(r N-\sum_{i=1}^{N} \frac{1+\sigma_{i}}{2}\right)$,

at a given quenched disorder $\left\{J_{i j k}\right\}$ and configuration $\underline{s}$. We introduced the indicator function

$$
\mathbb{I}_{\underline{\sigma}}(\underline{s})=\prod_{i=1}^{N}\left\{\frac{1-\sigma_{i}}{2}+\frac{1+\sigma_{i}}{2} \theta\left[u_{i}^{2}-\left(v_{i}+w_{i}\right)^{2}-\epsilon\right]\right\},
$$

returning one if and only if $\underline{\sigma}$ defines a SSC in the configuration $\underline{s}$. The $\theta(x)$ in Eq. (5) is the Heaviside function, returning one for $x>0$ and zero otherwise: its role is to select only those realizations $\underline{\sigma}$ for which the condition given by Eq. (3) holds. Finally, the Dirac delta function in Eq. (4) enforces the size of the clusters to be $r N$.

Equation (4) gives the logarithm of the number of SSC (entropy of the clusters) per spin in a given configuration $\underline{s}$. As we are interested in the number of SSC in a typical configuration, and assuming that $\mathcal{S}\left(r \mid \underline{s},\left\{J_{i j k}\right\}\right)$ is selfaveraging with respect to $\underline{s}$ and the quenched disorder $\left\{J_{i j k}\right\}$, we define

$$
\mathcal{S}_{\beta}(r)=\mathbb{E}_{J} \mathbb{E}_{\underline{s}}\left[\mathcal{S}\left(r \mid \underline{s},\left\{J_{i j k}\right\}\right)\right],
$$

where $\mathbb{E}_{J}$ denotes the average over the quenched disorder and $\mathbb{E}_{\underline{s}}$ is the average over the Boltzmann weight $Z^{-1} e^{-\beta H(\underline{s})}$. This is the central object of our computation, because the number of $\operatorname{SSC} \mathcal{N}_{\beta}(r)$ of size $r$ in a typical configuration at temperature $\beta^{-1}$ is given by

$$
\mathcal{N}_{\beta}(r)=\exp \left[N \mathcal{S}_{\beta}(r)\right] .
$$

The number of large clusters is expected to grow as $T$ decreases, signalling the slowing down of the dynamics.

To investigate the stability of SSC, we compute the distribution of the local fields acting on the internal spins. If we consider the SSC of size $r N$, the quantity of interest is the local fields acting on the in-cluster spins,

$$
P_{r}(h=\lambda)=\mathbb{E}_{J} \mathbb{E}_{\underline{s}} \mathbb{E}_{\underline{\sigma}}^{r}\left[\frac{1}{r N} \sum_{i} \frac{1+\sigma_{i}}{2} \delta\left(h_{i}-\lambda\right)\right],
$$

where $\mathbb{E}_{\underline{\sigma}}^{r}$ is the average over SSC of size $r$,

$$
\mathbb{E}_{\underline{\sigma}}^{r}\left[O\left(\underline{\sigma}, \underline{s},\left\{J_{i j k}\right\}\right)\right]=\frac{\sum_{\underline{\sigma}} \mathbb{I}_{\underline{\underline{\sigma}}}^{r}(\underline{s}) O\left(\underline{\sigma}, \underline{s},\left\{J_{i j k}\right\}\right)}{\sum_{\underline{\sigma}} \mathbb{I}_{\underline{\sigma}}^{r}(\underline{s})},
$$


and the auxiliary function $\mathbb{I}_{\underline{\sigma}}^{r}(\underline{s})$ is given by

$$
\mathbb{I}_{\underline{\sigma}}^{r}(\underline{s})=\mathbb{I}_{\underline{\sigma}}(\underline{s}) \delta\left(r N-\sum_{i=1}^{N} \frac{1+\sigma_{i}}{2}\right) .
$$

An SSC where many spins experience a strong field can be regarded as a cluster of slow-evolving spins, because the probability of spin flips decreases as the absolute value of the local field increases. The field $P_{r}(h)$ is supported primarily for small fields at high temperatures and large fields at low temperatures. As shown in the SM this distribution can be obtained from Eq. (6).

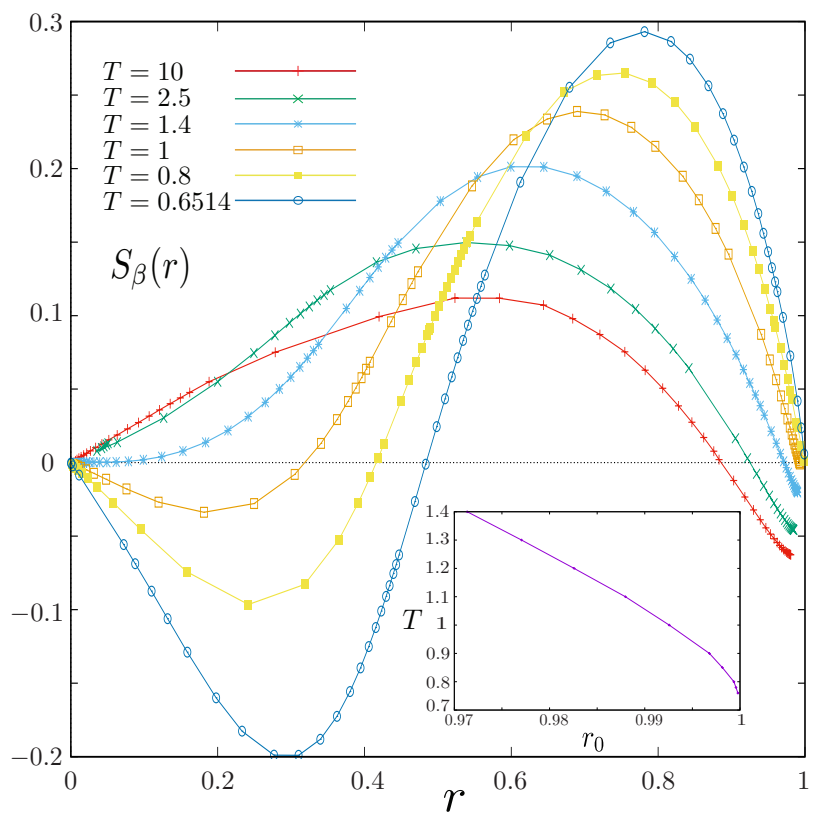

FIG. 1. Entropy of the SSC as a function of $r$, where $r N$ is the size of the self sustained cluster, for different temperatures. As the temperature decreases, we observe an increase in the number of large clusters (here, for $\epsilon=0$ ). Inset - We observe SSC for cluster size up to $r_{0} N$ which increases as $T$ decreases, approaching 1 for temperatures in the region between $T_{d} \sim$ 0.681 and $T_{T A P} \sim 0.764$.

Analysis: To average over the Boltzmann weights and the disorder and compute $\mathcal{S}_{\beta}(r)$ we will invoke the replica trick twice, once to replace the logarithm in Eq. (4) and once to account for the partition function in Eq. (6). The mathematical identities that we are going to use are:

$$
\log \sum_{\underline{\sigma}} \mathbb{I}_{\sigma}^{r}(\underline{s})=\lim _{m \rightarrow 0} \partial_{m} \sum_{\underline{\sigma}^{(1)} \ldots \underline{\sigma}^{(m)}} \prod_{\alpha=1}^{m} \mathbb{I}_{\underline{\sigma}^{(\alpha)}}^{r}\left(\underline{s}^{(1)}\right),
$$

where $\partial_{m}$ is the derivative with respect to $m, \underline{s}^{(1)}=\underline{s}$ and

$$
Z^{-1}=\lim _{n \rightarrow 0} \sum_{\underline{s}^{(2)} \ldots \underline{s}^{(n)}} \exp \left[-\beta \sum_{a=2}^{n} H\left(\underline{s}^{(a)}\right)\right] .
$$

Expressions are calculated for integers $n$ and $m$ values and then analytically continued to zero [3]. Greek and
Latin indices denote replicas of the $\sigma$ and $s$ variables, respectively. The details of the computation are discussed in the SM. In this work we employed a Replica Symmetric (RS) ansatz. In principle, averaging over configurations $\underline{s}$, one should invoke a more complex hierarchical ansatz [3] but the RS ansatz is valid for all temperatures higher than $T_{K}$, even in the dynamical region between $T_{K} \sim 0.652$ and $T_{d} \sim 0.681$ for reasons that can be traced back to the work of Franz and Parisi [36]. In fact, in this regime, the paramagnetic state is replaced by an exponential number of metastable states whose overlap is zero [37-39], from which a trivial Parisi function $P(q)=\delta(q)$ is obtained. We also employ an RS ansatz for the $\sigma$-related order parameters. This is just an approximation that is not always justified as explained below.

Results: We computed the entropy of SSC for different temperatures and values of $\epsilon$. While we do not observe abrupt changes in the entropy of the SSC, the number of large SSC increases and non-zero entropies appear for larger clusters when $T$ decreases, as shown in Fig. 1. A numerical analysis performed on small fully connected systems by sampling configurations from Monte-Carlo, confirm this description, as can be seen in Fig. 2. Details on the procedure employed for obtaining numerical results on small systems are provided in Section D of the SM.

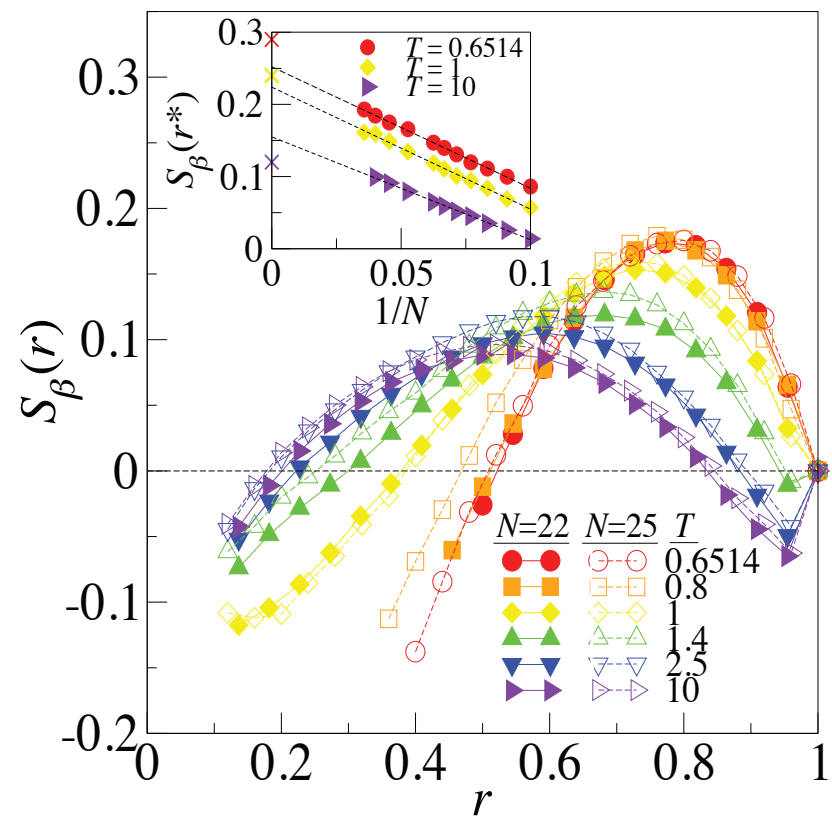

FIG. 2. The entropy of self-sustained clusters in small 3spin systems. The systems are first initialized with random coupling $J_{i j k}$ and are equilibrated for $5 \times 10^{4}$ Monte Carlo steps at temperature $T$ before sampling takes place for $1 \times 10^{3}$ steps. The number of SSC is then computed by exhaustive search over all $\sigma$ in systems with $N=22$, and by a random sample of $4 \times 10^{\frac{6}{6}}$ of all $\underline{\sigma}$ configurations in systems with $N=25$; each sampled spin configuration is weighed by the number of times it is sampled. The results are then averaged over 5 realizations of coupling disorders. 


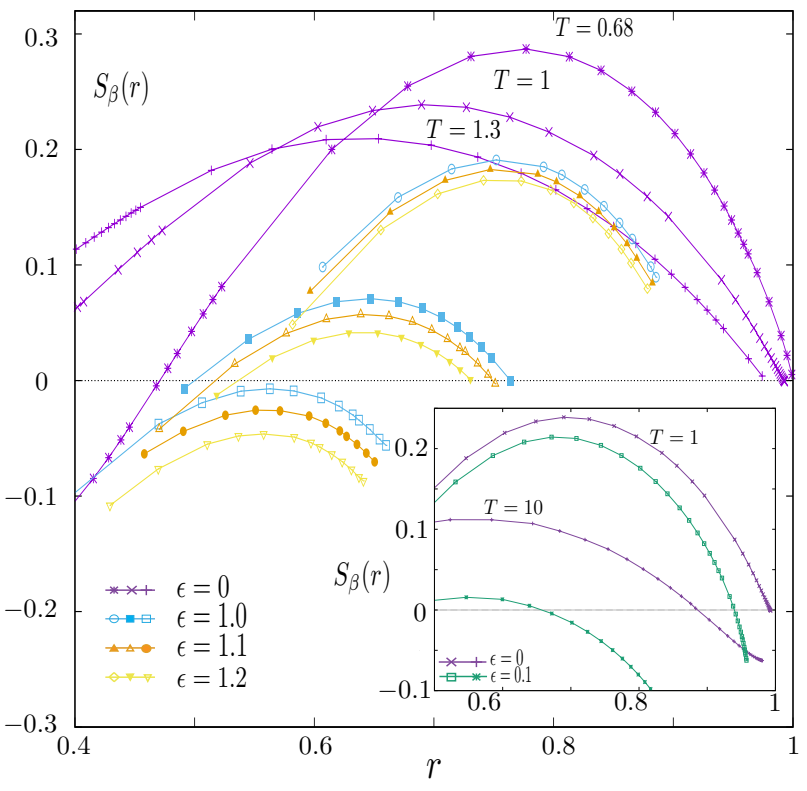

FIG. 3. Entropy of the SSC as a function of $r$, where $r N$ is the size of the self sustained cluster, for different temperatures and different $\epsilon$ values. The inset shows the effect of taking the same small $\epsilon$ at two different temperatures: it can be clearly seen that at $T=10$ it decimates the number of clusters but has relatively little effect at $T=1$.

This behavior has a simple interpretation in terms of the effect of random fluctuations on in-cluster spins. Each spin's internal field $u_{i}$ is aligned with the total field $h_{i}$ and, since fluctuations involving a finite number of spins do not result in macroscopic contributions to the difference between in- and out-cluster induced fields, the alignment between in-cluster and total fields is largely insensitive to fluctuations. In other words, in-cluster spins provide a reinforcement mechanism to one another through the in-cluster dominated field that compensates for random fluctuations, which is absent for out-cluster spins. When several SSC overlap, a competition between the influence of different SSC forming islands of constrained spins, emerges. SSC appear in sizes up to $r_{0} N$ depending on temperature as shown in the inset of Fig. 1, with $r_{0}$ approaching 1 as $T$ decreases. Because of numerical instabilities in solving saddle point equations, it is impossible to evaluate precisely the temperature at which $r_{0}$ approaches 1 . This temperature is supposed to be in the range $\left[T_{d}, T_{T A P}\right]$, where in this model $T_{T A P} \sim 0.764$, as obtained in the SM.

We also notice that there are regions where the SSC entropy is negative. In replica calculations this effect can be interpreted a manifestation of RS-breaking; notice that, as discussed above, this could only happen in the $\sigma$-related order parameters. This effect is not trivial and may be due to the complicated structure of the SSC solutions in a given configuration. This point requires a careful analysis which is beyond the scope of the current

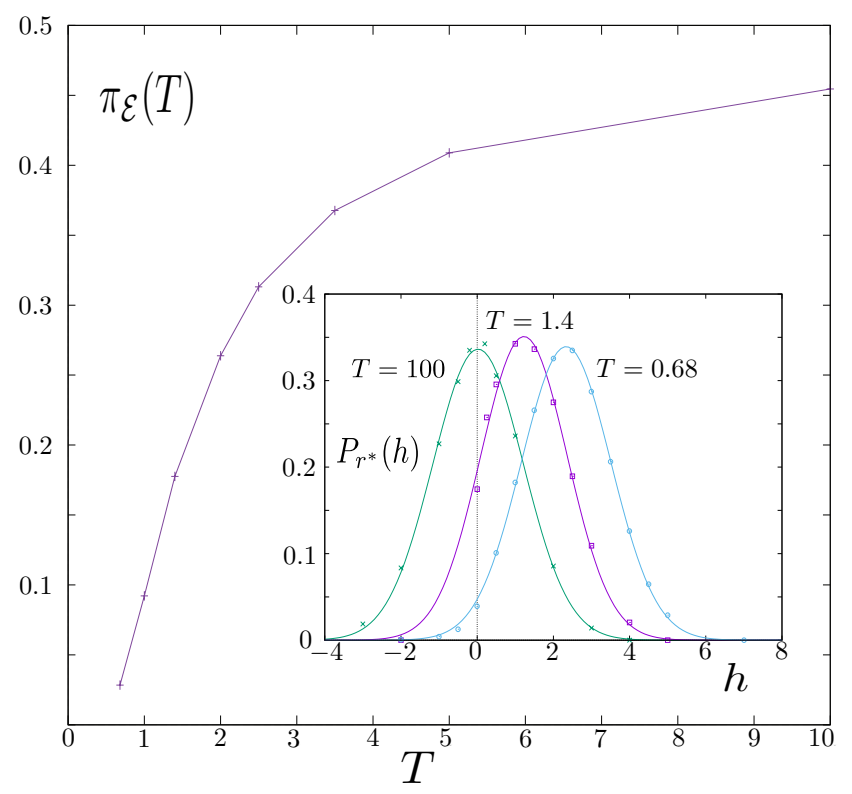

FIG. 4. Expected flip probability for spins in an SSC of size $r^{*} N$, where $r^{*}$ is the cluster-size value at which the entropy is maximum. Inset: $P_{r}(h)$ for three different temperatures.

analysis since here we focus on dominant peak values of the SSC entropy that are found to be positive. Therefore, in the following, we will concentrate only on regimes of positive SSC entropies.

The existence of SSC at high temperatures suggests that their existence does not trivially relate to slowevolving spins, which do not exist in this regime. Results obtained for different resilience parameter values $\epsilon$ (see Eq. (3)), shown in Fig. 3, exhibit a strong presence of low-field SSC at high temperatures. Firstly, we note that the absolute value of the local field experienced by internal spins is an increasing function of $\epsilon$. A slow-evolving spin is characterized by a strong field such that $\beta\left|h_{i}\right|$ is large. Thus, SSC can be associated with slow-evolving spins only if they exist for a sufficiently high $\epsilon$ and this argument suggests that $\epsilon$ should be an increasing function of $T$. For instance, the scaling $|h| \sim \sqrt{\epsilon}$ leads to $\epsilon \sim T^{2}$. Fig. 3 shows that as we increase $\epsilon$, fewer and fewer SSC exist with a much stronger effect exhibited at high temperatures, as demonstrated in the inset. Even a very small value of $\epsilon$ (e.g. $\epsilon=0.1$ ) decimates the number of SSC at high temperature (e.g. $T=10$ ) while strong clusters are unaffected at lower temperatures (e.g. $T=1$ ).

To verify the analytical picture obtained, we compute the entropy of SSC by exhaustive search or sampling in small 3-spin systems. As shown in Fig. 2, SSC exist at high temperature and the entropy of large SSC increases when the temperature $T$ decreases in small systems, in agreement with the analytical results shown in Fig. 1. On the other hand, SSC with small $r$ values may be absent in small system sizes due to finite-size effects as shown in 
Fig. 2, compared to the thermodynamic limit in Fig. 1. Despite the slightly different definition of SSC entropy employed in the numerical analysis of small systems (see Section D of SM for details), their behaviors match qualitatively with the analytical results as shown in Fig. 2 and its inset.

To gain a quantitative measure of how slow the incluster spins are, we computed the corresponding distribution of local fields $P_{r}(h)$ using Eqs. (8)-(10). We employed this distribution to compute the expected flip probability spins in an SSC of size $r^{*} N$

$$
\pi_{\mathcal{E}}(T)=\int d h P_{r^{*}}(h) \frac{e^{-\beta|h|}}{2 \cosh (\beta h)},
$$

where $r^{*}$ is the the value at which $S_{\beta}(r)$ is maximum. The expected probability $\pi_{\mathcal{E}}(T)$ rapidly decreases to zero as $T$ decreases, as shown in Fig. 4 . The inset shows $P_{r}^{*}(h)$ for different temperatures. The relation between SSC and slow-evolving spins in sparsely connected topologies will be further explored in a separate work.

The above results show that when the temperature decreases, SSC in the 3 -spin model increase in number, become more extensive in size, and are more stable against thermal fluctuations. Since these clusters are self-sustained, their stable existence slows down the system's dynamics towards equilibrium. In optimization problems, the presence of SSC would induce computational hardness since local algorithms will not escape states with SSC on the search for optimal solutions.

Summary: We proposed a theoretical framework to address the issue of slow-evolving variables in spin systems at the microscopic level based on the concept of SSC, which can be viewed as regions of interdependent mutually-stabilizing spins. As the temperature decreases, strong SSC emerge and encompass increasingly larger fraction of the system with inevitable conflicts between competing clusters. We provide new microscopic perspective on the dynamical slowing down observed in spin systems at low temperatures, complementing existing macroscopic understanding with the potential of providing new algorithmic optimization tools for hard computational problems through the destabilization of SSC in problems that can be mapped onto spin systems.

\section{Acknowledgements}

This work is supported by The Leverhulme Trust grant RPG-2013-48 and the Research Grants Council of Hong Kong (CHY, Grant No. 28300215, 18304316). We are grateful to Leticia Cugliandolo for pointing us to references [27-30] and for helpful comments. We would also like to thank Pierfrancesco Urbani for interesting discussions.
[1] D. Sherrington and S. Kirkpatrick. Physical Review Letters, 35(26):1792, 1975.

[2] G. Parisi. Physical Review Letters, 43(23):1754, 1979.

[3] M. Mézard, G. Parisi, and M.-A. Virasoro. Spin glass theory and beyond. World Scientific Publishing Co., Inc., Pergamon Press, 1990.

[4] G. Parisi. Complex systems. edited by J.-P. Bouchaud, M. Mézard and J. Dalibard. Elsevier, Les Houches, France, 2007.

[5] F. Zamponi. arxiv.org/abs/1008.4844.

[6] T. Castellani and A. Cavagna. Journal of Statistical Mechanics: Theory and Experiment, 2005(05):P05012, 2005.

[7] K. Binder and A. P. Young. Reviews of Modern physics, 58(4):801, 1986.

[8] T.R. Kirkpatrick and D. Thirumalai. Physical Review B, 36(10):5388, 1987.

[9] J.-P. Bouchaud, L.F. Cugliandolo, J. Kurchan, and M. Mezard. Spin glasses and random fields, pages 161$223,1998$.

[10] L. Leuzzi and T. M. Nieuwenhuizen. Thermodynamics of the glassy state. Taylor and Francis, 2007.

[11] W. Götze. Complex dynamics of glass-forming liquids: A mode-coupling theory, volume 143. OUP Oxford, 2008.

[12] R. Monasson. Complex systems. edited by J.-P. Bouchaud, M. Mézard and J. Dalibard. Elsevier, Les Houches, France, 2007.

[13] M. Mezard and A. Montanari. Information, physics, and computation. Oxford University Press, 2009.
[14] D.J. Thouless, P.W. Anderson, and R.G. Palmer. Philosophical Magazine, 35(3):593-601, 1977.

[15] R. Monasson. Physical review letters, 75(15):2847, 1995.

[16] A. Crisanti and H.-J. Sommers. Zeitschrift für Physik B Condensed Matter, 87(3):341-354, 1992.

[17] A. Crisanti, H. Horner, and H.-J. Sommers. Zeitschrift für Physik B Condensed Matter, 92(2):257-271, 1993.

[18] L.F. Cugliandolo and J. Kurchan. Physical Review Letters, 71(1):173, 1993.

[19] A. Barrat, R. Burioni, and M. Mézard. Journal of Physics A: Mathematical and General, 29(5):L81, 1996.

[20] A. Montanari and F. Ricci-Tersenghi. The European Physical Journal B-Condensed Matter and Complex Systems, 33(3):339-346, 2003.

[21] M. Mézard, F. Ricci-Tersenghi, and R. Zecchina. Journal of Statistical Physics, 111(3-4):505-533, 2003.

[22] F. Krzakała, A. Montanari, F. Ricci-Tersenghi, G. Semerjian, and L. Zdeborová. Proceedings of the National Academy of Sciences, 104(25):10318-10323, 2007.

[23] A. Montanari, F. Ricci-Tersenghi, and G. Semerjian. Journal of Statistical Mechanics: Theory and Experiment, 2008(04):P04004, 2008.

[24] D. Achlioptas and F. Ricci-Tersenghi. SIAM Journal on Computing, 39(1):260-280, 2009.

[25] G. Semerjian. Journal of Statistical Physics, 130(2):251293, 2008.

[26] A. Braunstein, L. Dall'Asta, G. Semerjian, and L. Zdeborová. Journal of Statistical Mechanics: Theory and Experiment, 2016(5):053401, 2016. 
[27] A. Barrat and R. Zecchina. Physical Review E, 59(2):R1299, 1999.

[28] F. Ricci-Tersenghi and R. Zecchina. Physical Review E, 62(6):R7567, 2000.

[29] F. Romá, S. Bustingorry, and P.M. Gleiser. Physical review letters, 96(16):167205, 2006.

[30] F. Romá, S. Bustingorry, and P.M. Gleiser. Physical Review B, 81(10):104412, 2010.

[31] F. Barahona, R. Maynard, R. Rammal, and J.P. Uhry. Morphology of ground states of two-dimensional frustration model. Journal of Physics A: Mathematical and General, 15(2):673, 1982.

[32] C.H. Yeung and D. Saad. Physical Review E, 88(3):032132, 2013.
[33] B. Derrida. Physical Review Letters, 45(2):79, 1980.

[34] A. Crisanti, L. Leuzzi, and T. Rizzo. Complexity in mean-field spin-glass models: Ising p-spin. Physical Review B, 71(9):094202, 2005.

[35] T. Rizzo. Physical Review E, 88(3):032135, 2013.

[36] S. Franz and G. Parisi. Journal de Physique I, 5(11):1401-1415, 1995.

[37] T.R. Kirkpatrick and D. Thirumalai. Physical Review Letters, 58(20):2091, 1987.

[38] J. Kurchan, G. Parisi, and M.-A. Virasoro. Journal de Physique I, 3(8):1819-1838, 1993.

[39] A. Crisanti and H.-J. Sommers. Journal de Physique I, $5(7): 805-813,1995$ 


\title{
Supplementary Material
}

Self-Sustained Clusters as Drivers of Computational Hardness in p-spin Models

\author{
Jacopo Rocchi* and David Saad ${ }^{\dagger}$ \\ Nonlinearity and Complexity Research Group, \\ Aston University, Birmingham B4 7ET, United Kingdom \\ Chi Ho Yeung ${ }^{\ddagger}$ \\ Department of Science and Environmental Studies, \\ The Education University of Hong Kong, Hong Kong
}

\footnotetext{
* j.rocchi@aston.ac.uk

$\dagger$ d.saad@aston.ac.uk

‡ chyeung@eduhk.hk
} 


\section{SUPPLEMENTARY INFORMATION}

In this section we first describe some aspects of the Ising p-spin model, and then we illustrate the computation of the entropy of SSC. The phenomenology of the p-spin is discussed presenting the Franz-Parisi potential and the computation of the complexity of the system. While the first method is very interesting by its own right, it is also very instructive for our purposes because it allows to discuss some more technical issues that will be useful in the computation of the entropy of SSC. The second method gives a different perspective on the emergence of TAP states in this model, and provides an estimation of the temperature, $T_{T A P}$, at which such states appear. The two approaches are independent and complementary. More aspects of the rich phenomenology of the model can be found in [33, 34]. Here we describe only the case $p=3$.

\section{A. Franz-Parisi potential}

The Franz-Parisi potential [35] can be defined by introducing the free energy per spin of a system that is constrained to have overlap $q$ with a reference configuration $\underline{s}$

$$
-N \beta F_{\underline{s},\left\{J_{i j k}\right\}}(q)=\log \sum_{\underline{\sigma}} e^{-\beta H(\underline{\sigma})} \delta\left(N q-\sum_{i} \sigma_{i} s_{i}\right) .
$$

This quantity depends on $\underline{s}$ and $\left\{J_{i j k}\right\}$ and in order to get rid of these dependancies we assume self-averaging properties on both. In other words, we compute

$$
V(q)=\mathbb{E}_{J} \mathbb{E}_{\underline{s}}\left[F_{\underline{s},\left\{J_{i j k}\right\}}(q)-F\right]
$$

where, as in the Main Text, $\mathbb{E}_{J}$ denotes the average over disorder and $\mathbb{E}_{\underline{s}}$ the average over the Boltzmann weight. The Hamiltonian of the model is defined in eq. 1 and the quenched disorder is such that $J_{i j k}$ are i.i.d. Gaussian random variables, with mean 0 and variance $p ! /\left(2 N^{p-1}\right)$, where $N$ is the number of spins. This quantity is the large deviation function of the probability to observe an overlap equal to $q$ between two configurations extracted from the equilibrium (Boltzmann) distribution, i.e. the Parisi function $P(q)$, and one of the main reasons to study $V(q)$ is that it contains information about the dynamical transition $T_{d}$ that is actually missing in both $P(q)$ and $F$. In order to compute $V(q)$ we need to use the replica trick twice. This can be done invoking eq. (12) to deal with the Boltzmann weight, and the 
identity

$$
-N \beta F_{\underline{s},\left\{J_{i j k}\right\}}(q)=\lim _{m \rightarrow 0} \partial_{m} \sum_{\{\underline{\sigma}\}} e^{-\beta \sum_{\alpha=1}^{m} H\left[\underline{\sigma}^{(\alpha)}\right]} \prod_{\alpha=1}^{m} \delta\left(N q-\sum_{i} \sigma_{i}^{(\alpha)} s_{i}^{(1)}\right)
$$

where $\{\underline{\sigma}\}=\left\{\underline{\sigma}^{(1)} \ldots \underline{s}^{(m)}\right\}$, to deal with logarithm inside the averages. It is useful to introduce the order parameters

$$
\begin{aligned}
Q_{s s}^{a b} & =\frac{1}{N} \sum_{i} s_{i}^{(a)} s_{i}^{(b)} \\
Q_{\sigma \sigma}^{\alpha \beta} & =\frac{1}{N} \sum_{i} \sigma_{i}^{(\alpha)} \sigma_{i}^{(\beta)} \\
Q_{s \sigma}^{a \alpha} & =\frac{1}{N} \sum_{i} s_{i}^{(a)} \sigma_{i}^{(\alpha)},
\end{aligned}
$$

and the corresponding conjugate ones (order parameters with hats) by using the integral representation of the Dirac delta function, here illustrated for $Q_{s s}$ :

$$
1=\prod_{a<b} \int d Q_{\sigma \sigma}^{a b} \frac{d \hat{Q}_{\sigma \sigma}^{a b}}{2 \pi} e^{-i \hat{Q}_{\sigma \sigma}^{a b}\left(N Q_{\sigma \sigma}^{a b}-\sum_{i=1}^{N} s_{i}^{(a)} s_{i}^{(b)}\right)} .
$$

In the following we adopt an RS ansatz, that is supposed to hold $\forall T>T_{K}$ [35]. The order parameters $Q_{s s}$ and $Q_{\sigma \sigma}$ are the off diagonal terms of the matrices defined in eq. (17) and (18). The delta function in eq. (14) sets $Q_{s \sigma}^{1 \alpha}=q, \forall \alpha$, and thus, since the first row of this matrix is $\alpha$ independent, we also set $Q_{s \sigma}^{a \alpha}=P^{a}, a \neq 1, \forall \alpha$ and in the RS ansatz we set $P^{a}=P, a \neq 1$. Similarly, we set $\hat{Q}_{s \sigma}^{1 \alpha}=\hat{q}, \forall \alpha$ and $\hat{Q}_{s \sigma}^{a \alpha}=\hat{P}, a \neq 1, \forall \alpha$. These manipulations lead to

$$
-\beta V(q)=\beta^{2}\left(\frac{1}{4}-\frac{1}{4} Q_{\sigma \sigma}^{3}+\frac{1}{2} q^{3}-\frac{1}{2} P^{3}\right)+\frac{1}{2}\left(Q_{\sigma \sigma}-1\right) \hat{Q}_{\sigma \sigma}-\hat{q} q+\hat{P} P+\mathcal{I}
$$

where the integral $\mathcal{I}$ is equal to $\mathcal{I}=\int D \underline{z} h\left(z_{1}, z_{2}, \hat{q}, \hat{P}\right)$ and

$$
h\left(z_{1}, z_{2}, \hat{q}, \hat{P}\right)=\frac{\log 2 \cosh \left(z_{2}+(\hat{q}-\hat{P})\right) e^{z_{1}}+\log 2 \cosh \left(z_{2}-(\hat{q}-\hat{P})\right) e^{-z_{1}}}{2 \cosh z_{1}}
$$

and the Gaussian measure $D \underline{z}$ is

$$
D \underline{z}=\sqrt{\frac{\operatorname{det}(U)^{-1}}{(2 \pi)^{2}}} \prod_{k=1}^{2} d z_{k} \exp \left\{-\frac{1}{2^{2}} \underline{z}^{T} U^{-1} \underline{z}\right\}, \quad U=\left(\begin{array}{cc}
\hat{Q}_{s s} & \hat{P} \\
\hat{P} & \hat{Q}_{\sigma \sigma}
\end{array}\right) .
$$


The saddle point sets the other order parameters to

$$
\begin{gathered}
\hat{Q}_{s s}=\frac{3}{2} Q_{s s}^{2} \beta^{2}, \\
\hat{Q}_{\sigma \sigma}=\frac{3}{2} Q_{\sigma \sigma}^{2} \beta^{2}, \\
\hat{P}=\frac{3}{2} P^{2} \beta^{2},
\end{gathered}
$$

while the original order parameters are set to

$$
\begin{gathered}
\frac{1}{2} Q_{s s}-\frac{1}{2}+\int D \underline{z}\left[\frac{1}{2} z_{1}^{2} \hat{Q}_{s s}^{-2}-\frac{1}{2} \hat{Q}_{s s}^{-1}\right] \log 2 \cosh z_{1}=0 \\
\frac{1}{2} Q_{\sigma \sigma}-\frac{1}{2}+\int D \underline{z}\left[\frac{1}{2}\left(\sum_{l}\left[U^{-1}\right]_{2 l} z_{l}\right)^{2}-\frac{1}{2}\left[U^{-1}\right]_{22}\right] h\left(z_{1}, z_{2}, \hat{q}, \hat{P}\right)=0 \\
P+\int D \underline{z}\left[\sum_{l p}\left[U^{-1}\right]_{1 l}\left[U^{-1}\right]_{2 p} z_{l} z_{p}-\left[U^{-1}\right]_{12}\right] h\left(z_{1}, z_{2}, \hat{q}, \hat{P}\right)=0 \\
q=\int D \underline{z}\left[\tanh \left(z_{2}+(\hat{q}-\hat{P})\right) e^{z_{1}}-\tanh \left(z_{2}-(\hat{q}-\hat{P})\right) e^{-z_{1}}\right]\left[2 \cosh z_{1}\right]^{-1} .
\end{gathered}
$$

Eq. (27) is independent of $P, Q_{\sigma \sigma}$ and their conjugate order parameters because the $\underline{s}$ system is unaffected by the computation of the constrained free energy in eq. (14). Moreover, eq. (30) gives the value of the overlap $q$, on which we did not have to optimize, as a function of all the others, on which we had to. Before solving these equations, we notice that as long as $T>T_{K}, Q_{s s}=\hat{Q}_{s s}=0$ and this condition lead to $P=\hat{P}=0$. Thus, rather than solving a system of equations, we end up with solving a single equation,

$$
\hat{Q}_{\sigma \sigma}=\frac{1}{2} \int D t\left[\tanh ^{2}\left(\hat{q}-\sqrt{\hat{Q}_{\sigma \sigma}} t\right)+\tanh ^{2}\left(\hat{q}+\sqrt{\hat{Q}_{\sigma \sigma}} t\right)\right]
$$

and, at the end, we only need to compute the corresponding value for $q$, given by

$$
q=\frac{1}{2} \int D t\left[\tanh \left(\hat{q}-\sqrt{\hat{Q}_{\sigma \sigma}} t\right)+\tanh \left(\hat{q}+\sqrt{\hat{Q}_{\sigma \sigma}} t\right)\right]
$$

where $\hat{Q}_{\sigma \sigma}$ is still given by eq. $(25)$ and $D t=\mathcal{N}(0,1)$ is a Normal Gaussian distribution with mean 0 and variance 1 . Moreover, thanks to this simplification, eq. (21) becomes

$$
-\beta V(q)=\beta^{2}\left(\frac{1}{4}-\frac{1}{4} Q_{\sigma \sigma}^{3}+\frac{1}{2} q^{3}\right)+\frac{1}{2}\left(Q_{\sigma \sigma}-1\right) \hat{Q}_{\sigma \sigma}-\hat{q} q+\mathcal{I}^{\prime}
$$

where

$$
\mathcal{I}^{\prime}=\frac{1}{2} \int D t\left[\log 2 \cosh \left(\hat{q}+\sqrt{\hat{Q}_{\sigma \sigma} t}\right)+\log 2 \cosh \left(\hat{q}-\sqrt{\hat{Q}_{\sigma \sigma}} t\right)\right] .
$$


We can solve eq. (31) for each $\{\beta, \hat{q}\}$ by iteration. Finally, plugging the solution in eq. (33) and eq. (32) we obtain a value for $V(q)$. The results can be seen in Fig. (5). The dynamical temperature $T_{d}$ is defined as the temperature at which the potential develops a local minimum at $q=q^{*}$ while the Kauzmann temperature $T_{K}$ as the temperature at which the local minimum becomes a global one. We see that $T_{d} \sim 0.681$ and $T_{K} \sim 0.652$.

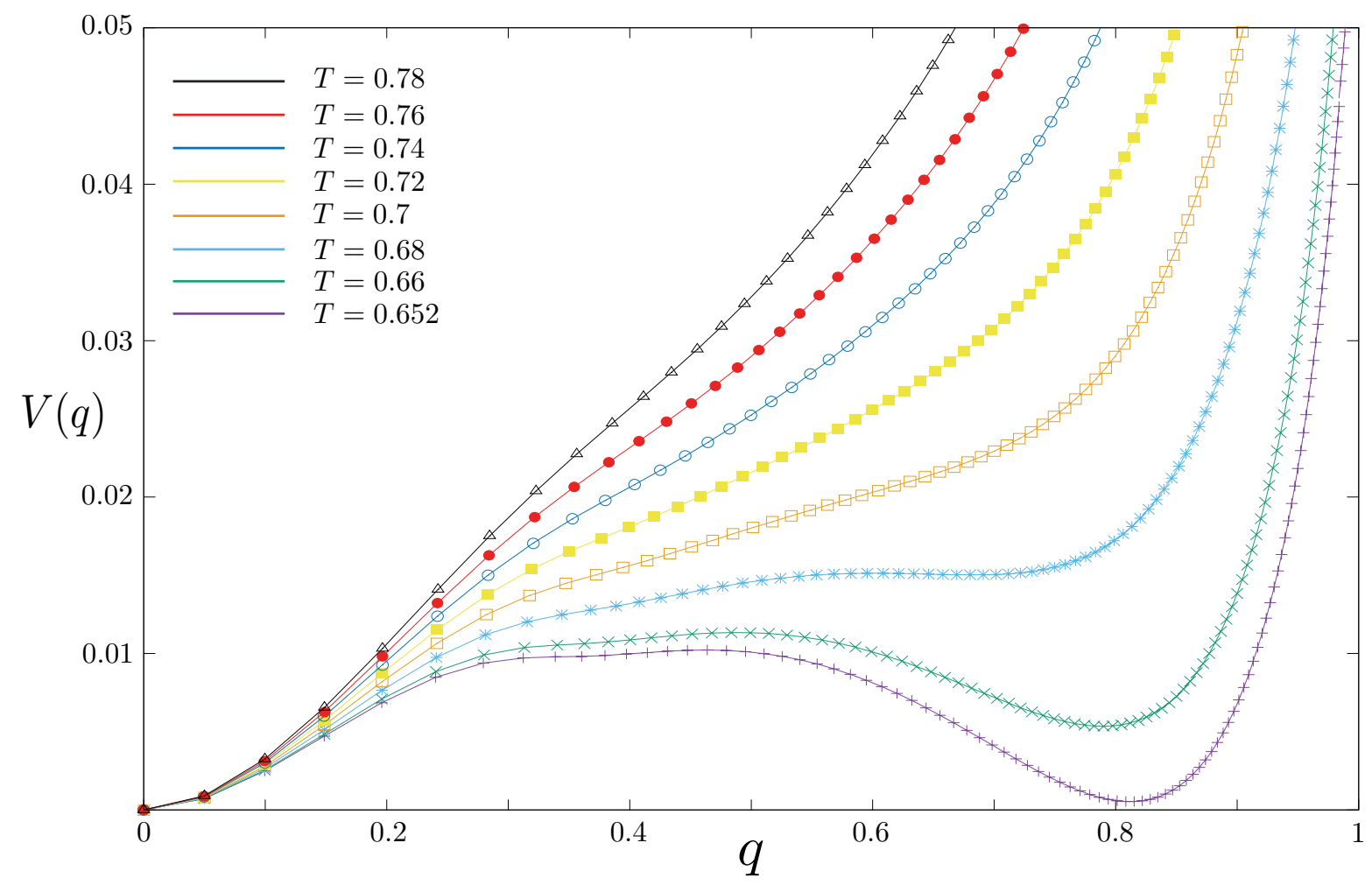

FIG. 5. Franz-Parisi potential of the Ising $3-\operatorname{spin}$. The potential has only one minimum at $q=0$ in the high temperature phase but as $T \rightarrow T_{d} \sim 0.681$ a metastable minimum appear at $q^{*}>0$. This second minimum becomes the global one for $T<T_{K} \sim 0.652$.

\section{B. Complexity}

The computation of the complexity of the model offers a different perspective. The name complexity denotes the entropy of the number of metastable states that dominate the Boltzmann weight in the dynamical phase. It can be computed by solving the TAP equations [13] of the model, as done in [33], or by counting the number of pure states (or TAP states) of the system. A detailed description of pure states can be found in [5]. They 
can be defined as measures on the configuration space with vanishing connected correlation functions between far degrees of freedom. This notion is intuitively related to equilibrium states, where the response function, at long distance, vanishes. Since in mean field model there is no notion of distance, pure states have to be such that

$$
P^{\omega}\left(s_{1}, \ldots, s_{N}\right)=\prod_{i=1}^{N} p^{\omega}\left(s_{i}\right), \quad p^{\omega}\left(s_{i}\right)=\frac{1+s_{i} m_{i}^{\omega}}{2}
$$

where $\omega$ identifies one of such states. In other words, in mean field models, pure states are such that all the connected correlation functions vanish. At high temperatures, only one such state exist, the paramagnetic state, where $m_{i}=0, \forall i$. As $T$ decreases, new states emerge. In this situation, the partition function can be decomposed in the following way

$$
Z=\sum_{\underline{s}} e^{-\beta H(\underline{s})}=\sum_{\omega} e^{-\beta N f_{\omega}},
$$

where $f_{\omega}$ denotes the internal free energy of the state $\omega$. In models without quenched disorder, when several pure states exist, they can be selected by introducing an external vanishing field. Unfortunately, spin glasses do not allow for a similar procedure because it is an unfeasible task to slightly perturb, locally, each spin in the correct direction. In order to solve this issue, Monasson [14] introduced the method of coupled replicas: replicas act as teachers for each other, and they all end up in the same pure state. Thus the partition function of $m$ coupled replicas ( $m$-system from now on) can be written as

$$
Z_{m}=\sum_{\omega} e^{-\beta m N f_{\omega}}=\int_{f_{m}}^{f_{t h}} d f \sum_{\omega} \delta\left(f-f_{\omega}\right) e^{-\beta m N f}=\int_{f_{m}}^{f_{t h}} d f e^{N[-\beta m f+\Sigma(f)]}
$$

where $f_{m}$ and $f_{t h}$ define the limits where pure states can be found and $\Sigma(f(m, T))$ is, by definition, the complexity of the $m$-system. Eventually, we will be interested in the limit $m \rightarrow 1$. This integral can be computed with a saddle point method and gives

$$
\Phi(m, T)=-\frac{T}{N} \mathbf{E}_{J} \log Z_{m}=m f^{*}(m, T)-T \Sigma\left(f^{*}(m, T)\right) .
$$

$f^{*}(m, T)$ is the free energy of the pure states of the $m$-system that dominates the Boltzmann weight at temperature $T$. Both $f^{*}(m, T)$ and $\Sigma\left(f^{*}(m, T)\right)$ can be found by differentiating $\Phi(m, T)$ :

$$
\begin{gathered}
\Sigma\left(f^{*}(m, T)\right)=m^{2} \frac{\partial\left[m^{-1} \beta \Phi(m, T)\right]}{\partial m} \\
f^{*}(m, T)=\frac{\partial \Phi(m, T)}{\partial m}
\end{gathered}
$$


and thus $m$ can be used as a dummy variable to compute numerically $\Sigma(f)$, which is the entropy of the pure states with free energy equal to $f$. This is a general protocol that can be carried out in every model. All we need to do is computing $\Phi(m, T)$ for which we need to use replicas:

$$
\Phi(m, T)=-\frac{T}{N} \mathbf{E}_{J} \log Z_{m}=-\frac{T}{N} \lim _{n \rightarrow 0} \partial_{n} \mathbf{E}_{J}\left(Z_{m}\right)^{n}
$$

Introducing the integral representation illustrated in (20), we end up dealing with order parameters $Q^{a b}$ and $\hat{Q}^{a b}$ that now live in a $m \times n$ dimensional space. These matrices contain $n$ groups of $m$-coupled replicas: it is thus natural to employ a $1-\mathrm{RSB}$ ansatz where the off-diagonal elements indexed by $\{a, b\}$ are zero for $a, b$ not in the same block, and take a positive value for $\{a, b\}$ in the same block. These manipulation lead to

$$
\Phi\left(m, \hat{Q}_{s s}, Q_{s s}, T\right)=-T m\left[-\frac{(m-1)}{2} \hat{Q}_{s s} Q_{s s}+\frac{\beta^{2}}{4}\left[(m-1) Q_{s s}^{3}+1\right]+m^{-1} \phi-\frac{1}{2} \hat{Q}_{s s}\right]
$$

where

$$
\phi=\log \int D t\left[2 \cosh \left(\sqrt{Q_{s s}} t\right)\right]^{m}
$$

and $\hat{Q}_{s s}$ and $Q_{s s}$ are fixed from the saddle point equations

$$
Q_{s s}=\frac{2}{m-1}\left\{-\frac{1}{2}+\frac{\sqrt{\hat{Q}_{s s}^{-1}}}{2} \frac{\int D t t\left[2 \cosh \sqrt{\hat{Q}_{s s} t}\right]^{m} \tanh \left(\sqrt{\hat{Q}_{s s}} t\right)}{\int D t\left[2 \cosh \sqrt{\hat{Q}_{s s}} t\right]^{m}}\right\}
$$

with $\hat{Q}_{s s}$ given by eq. (24). Eq. (44) has three solutions but we are interested only in the largest one. This is because, at a given $m>1$ and $T, \Phi\left(m, \hat{Q}_{s s}=3 \beta^{2} Q_{s s}^{2} / 2, Q_{s s}, T\right)$ has three stationary points as a function of $Q_{s s}$, the smallest one $\left(Q_{s s}=0\right)$ and the largest one $\left(Q_{s s}=q^{*}\right)$ being minima and the intermediate one being a maximum. These two values correspond to the overlap of different replicas in the $m$-system. As $m \rightarrow 1^{+}$the two minima are degenerate but, because of the coupling among replicas, $q^{*}$ has to be preferred. Using eq. (42) in eq. (39) and eq. (40) we finally obtain

$$
\begin{gathered}
\Sigma\left(f^{*}(m, T)\right)=m^{2}\left[\frac{\hat{Q}_{s s} Q_{s s}}{2}-\frac{\beta^{2}}{4} Q_{s s}^{3}+m^{-2} \phi-m^{-1} \partial_{m} \phi\right] \\
f^{*}(m, T)=(-T)\left[-\frac{m-1}{2} \hat{Q}_{s s} Q_{s s}+\frac{\beta^{2}}{4}\left[(m-1) Q_{s s}^{3}+1\right]+m^{-1} \phi-\frac{1}{2} \hat{Q}_{s s}\right]+\frac{T}{m} \Sigma\left(f^{*}(m, T)\right)
\end{gathered}
$$


that can be used to compute $\Sigma(f)$ at different temperatures by using $m$ as a parameter. This approach provides a phase diagram in the $m-T$ plane shown in Fig. 6, where three lines can be identified. $m^{*}(T)$ is the line below which eq. (44) has no solution with $q^{*}>0$. Below this line, the system in the paramagnetic state and the intersection between this line and the line $m=1$ identifies the dynamical transition. $m_{s}(T)$ is defined as the line where the complexity vanishes. Its intersection with the line $m=1$ identifies $T_{K}$. The intermediate line, named $m_{t h}(T)$ defines a non-physical region in this plane, contained between $m_{t h}(T)$ and $m^{*}(T)$. This region is called non-physical because the effect of increasing $m$ in the ratio $m / T$ is the same as that of decreasing $T$. When $T$ decreases, the free energy decreases as well, and thus regions where the free energy is an increasing function of $m$ are non-physical. $m_{t h}(T)$ crosses and merge with $m_{d}(T)$ for $T>T_{d}$. Pictorially, this behaviour can be described by observing that a large $m$ has the same effect of a small $T$. Thus, at a given $T$, the region where $f(m, T)$ and $\Sigma(f(m, T))$ grow with $m$ is un-physical (since they are both expected to decrease as we decrease $T$ ). This argument also suggests that $m$ can be effectively used as parameter that is able to probe non-equilibrium TAP-states. In fact, by definition, in the region between $m_{t h}(T)$ and $m_{s}(T)$ the complexity is positive and we see that this region extends for $T>T_{d}$, where the original system (that can be recovered for $m \rightarrow 1$ ) is in the paramagnetic phase. By the way it shrinks to zero as $T$ grows and the temperature at which $m_{s}(T)$ and $m_{t h}(T)$ merge is called $T_{T A P}$. This is the temperature at which TAP states forms as non-equilibrium states and its value in this model is $T_{T A P} \sim 0.764$.

\section{Entropy of Self-Sustained Clusters}

In this section we describe the computation of the entropy of SSC. Similarly to the computation of the Franz-Parisi potential described above, replicas are introduced to deal with the the logarithm in eq. (4) and with the Boltzmann weigh in eq. (6). Moreover, in order to be consistent with the notation used in the Main Text and in the computation of the Franz-Parisi potential, we denote by $\sigma$ the "internal" variables used to define clusters, and by $s$ the "external" variables referring to the spin of the configuration $\underline{s}$ extracted from the Boltzmann weight. In order to deal with the SSC membership condition, stated in eq. (3), let us repeat the definition of the local field, already given in eq. (2), and introduce the 


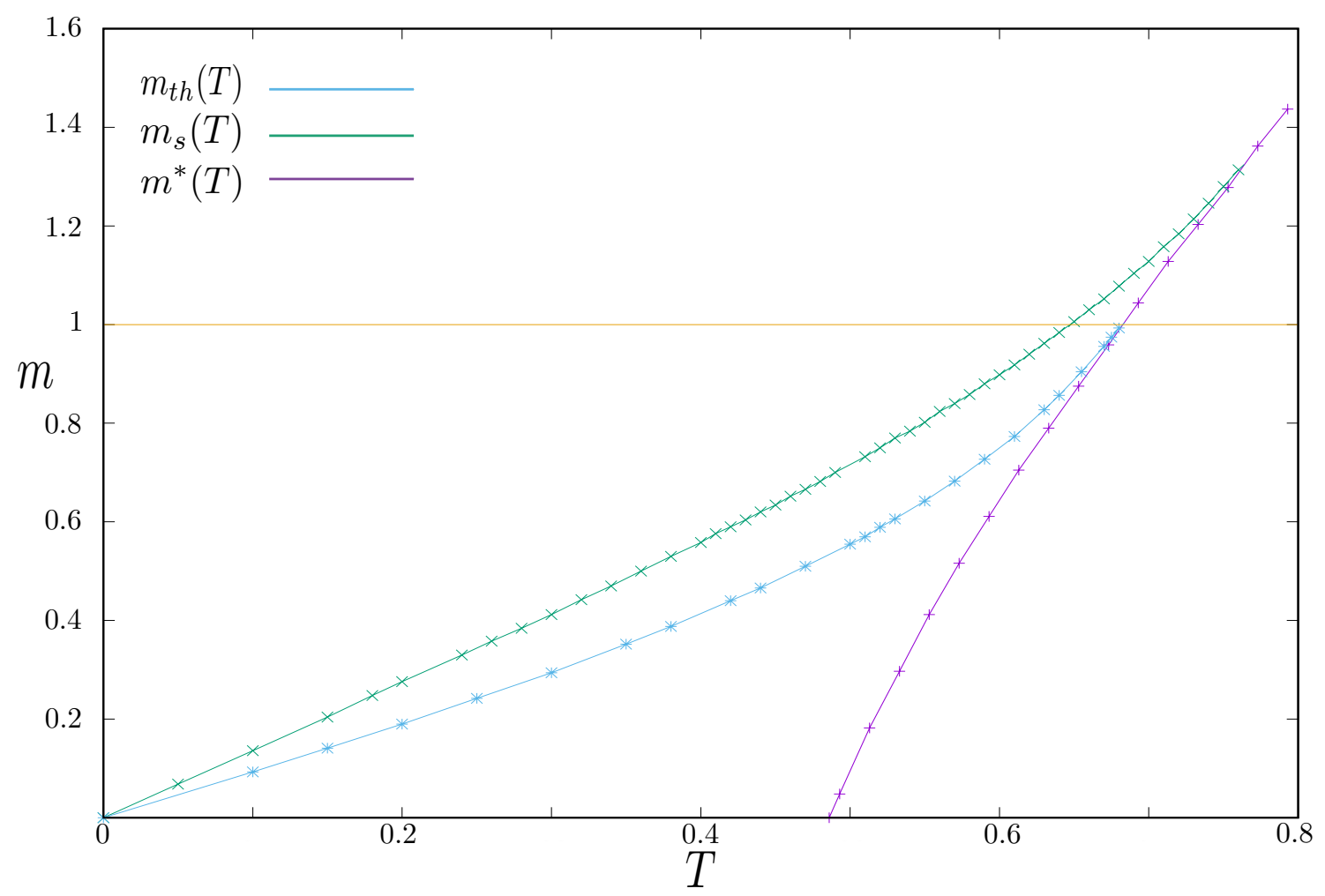

FIG. 6. Phase diagram of the Ising $3-$ spin in the $m-T$ plane discussed in the text. $T_{K} \sim 0.652$ is defined by the intersection between $m=1$ and $m_{s}(T) . T_{d} \sim 0.681$ is defined by the intersection between $m=1$ and $m^{*}(T) \cdot m_{t h}(T)$ and $m^{*}(T)$ merge for $T>T_{d}$. $T_{T A P} \sim 0.764$ is defined from the intersection between $m_{s}(T)$ and $m_{t h(T)}$.

two following replicated fields

$$
\begin{aligned}
h_{i} & =\frac{1}{2} \sum_{j, k} J_{i j k} s_{j} s_{k} \\
\eta_{i}^{(\alpha)} & =\frac{1}{2} \sum_{j, k} J_{i j k} s_{j}^{(1)} s_{k}^{(1)} \sigma_{j}^{(\alpha)} \sigma_{k}^{(\alpha)}, \\
\mu_{i}^{(\alpha)} & =\sum_{j, k} J_{i j k} s_{j}^{(1)} s_{k}^{(1)} \sigma_{j}^{(\alpha)} .
\end{aligned}
$$

For the sake of convenience, we report here the definition of entropy of SSC that we are going to compute in the following. $\mathcal{S}_{\beta}(r)$ is defined by

$$
\mathcal{S}_{\beta}(r)=\mathbb{E}_{J} \mathbb{E}_{\underline{s}}\left[\mathcal{S}\left(r \mid \underline{s},\left\{J_{i j k}\right\}\right)\right]
$$


where $\mathcal{S}\left(r \mid \underline{s},\left\{J_{i j k}\right\}\right)$ denotes the entropy of SSC in a given configuration $\underline{s}$ and at a given quenched disorder realisation $\left\{J_{i j k}\right\}$,

$$
\mathcal{S}\left(r \mid \underline{s},\left\{J_{i j k}\right\}\right)=N^{-1} \log \sum_{\underline{\sigma}} \mathbb{I}_{\underline{\sigma}}(\underline{s}) \delta\left(r N-\sum_{i=1}^{N} \frac{1+\sigma_{i}}{2}\right) .
$$

Thanks to the integral representation of the delta function, used to enforce the definitions of eq. (2), (48) and (49), eq. (50) can be written as

$$
N \mathcal{S}_{\beta}(r)=\lim _{n \rightarrow 0} \lim _{m \rightarrow 0} \mathbb{E}_{J}\left[\partial_{m} \mathcal{N}_{n m}\right]
$$

where the replicated quantity $\mathcal{N}_{n m}$ is given by

$$
\begin{aligned}
\mathcal{N}_{n m}= & \sum_{\{\underline{s}\}} \sum_{\{\underline{\sigma}\}} \int \mathcal{D} \mathcal{B \mathcal { B }} \exp \left\{-i \sum_{i} \hat{h}_{i} h_{i}-i \sum_{\alpha, i} \hat{\eta}_{i}^{(\alpha)} \eta_{i}^{(\alpha)}-i \sum_{\alpha, i} \hat{\mu}_{i}^{(\alpha)} \mu_{i}^{(\alpha)}\right\} \\
& \times \exp \left\{+\sum_{\alpha} \sum_{i j k} \frac{i \hat{\eta}_{i}^{(\alpha)}}{2} J_{i j k} s_{j}^{(1)} s_{k}^{(1)} \sigma_{j}^{(\alpha)} \sigma_{k}^{(\alpha)}+\sum_{\alpha} \sum_{i j k} i \hat{\mu}_{i}^{(\alpha)} J_{i j k} s_{j}^{(1)} s_{k}^{(1)} \sigma_{j}^{(\alpha)}\right\} \\
& \times \exp \left\{\sum_{i j k} \frac{i \hat{h}_{i}}{2} J_{i j k} s_{j}^{(1)} s_{k}^{(1)}\right\} \exp \left[-\beta \sum_{a=1}^{n} H\left(\underline{s}^{(a)}\right)\right] \prod_{\alpha=1}^{m} \underline{I}_{\underline{\sigma}^{(\alpha)}}\left(\underline{s}^{(1)}\right) \\
\mathbb{I}_{\underline{\sigma}^{(\alpha)}}^{r}\left(\underline{s}^{(1)}\right)= & \prod_{i=1}^{N}\left\{\frac{1-\sigma_{i}^{(\alpha)}}{2}+\frac{1+\sigma_{i}^{(\alpha)}}{2} \theta\left[\left(\mu_{i}^{(\alpha)}+\eta_{i}^{(\alpha)}-h_{i}\right) h_{i}-\epsilon\right]\right\} \delta\left(r N-\sum_{i=1}^{N} \frac{1+\sigma_{i}^{(\alpha)}}{2}\right)
\end{aligned}
$$

and we introduced the notation

$$
\mathcal{D B \mathcal { B }}=\prod_{i} \frac{d \hat{h}_{i} d h_{i}}{2 \pi} \prod_{i, \alpha} \frac{d \hat{\eta}_{i}^{(\alpha)} d \eta_{i}^{(\alpha)}}{2 \pi} \prod_{i, \alpha} \frac{d \hat{\mu}_{i}^{(\alpha)} d \mu_{i}^{(\alpha)}}{2 \pi}
$$

and the short-hand notation $\{\underline{s}\}$ for $\left\{\underline{s}^{(1)} \ldots \underline{s}^{(n)}\right\},\{\underline{\sigma}\}$ for $\left\{\underline{\sigma}^{(1)} \ldots \underline{s}^{(m)}\right\}$. The term $h_{i}$ contained in the argument of the Heaviside function in Eq. (54) does not have any replica index because it only depends on the configuration $\underline{s}=\underline{s}^{(1)}$. The effective fields $h_{i}, \eta_{i}^{(\alpha)}$ and $\mu_{i}^{(\alpha)}$ are used to make the argument of the Heaviside function disorder independent, so that the quenched average can be performed more easily. This average leads to the definition of several order parameters that we list in Table I. We also need to use the order parameters defined in eq. (17) and eq. (18). The last order parameter $Q_{s s \sigma}^{1 a \alpha}$ is equal to $m_{\sigma}$ for $a=1$, so we need to define it only for $a \neq 1$. Similarly to what has been done in eq. (20), introducing 
TABLE I. Order parameters introduced in the computation of the entropy of SSC. We will also make use of the order parameters defined in eq. (17) and eq. (18).

\begin{tabular}{lll}
\hline$Q_{\hat{\eta} \hat{\eta}}^{\alpha \beta}=-\frac{1}{N} \sum_{i} \hat{\eta}_{i}^{(\alpha)} \hat{\eta}_{i}^{(\beta)}$ & $Q_{\hat{\mu} \hat{\mu}}^{\alpha \beta}=-\frac{1}{N} \sum_{i} \hat{\mu}_{i}^{(\alpha)} \hat{\mu}_{i}^{(\beta)}$ & $Q_{\hat{\eta} \hat{\mu}}^{\alpha \beta}=-\frac{1}{N} \sum_{i} \hat{\eta}_{i}^{(\alpha)} \hat{\mu}_{i}^{(\beta)}$ \\
$C_{\hat{h} s \sigma}^{11 \alpha}=\frac{i}{N} \sum_{i} \hat{h}_{i} s_{i}^{(1)} \sigma_{i}^{(\alpha)}$ & $Q_{\hat{\eta} s \sigma}^{\alpha 1 \beta}=\frac{i}{N} \sum_{i} \hat{\eta}_{i}^{(\alpha)} s_{i}^{(1)} \sigma_{i}^{(\beta)}$ & $Q_{\hat{\mu} s \sigma}^{\alpha 1 \beta}=\frac{i}{N} \sum_{i} \hat{\mu}_{i}^{(\alpha)} s_{i}^{(1)} \sigma_{i}^{(\beta)}$ \\
$Q_{\hat{h} s}^{1 a}=\frac{i}{N} \sum_{i} \hat{h}_{i} s_{i}^{(a)}$ & $Q_{\hat{\eta} s}^{\alpha a}=\frac{i}{N} \sum_{i} \hat{\eta}_{i}^{(\alpha)} s_{i}^{(a)}$ & $Q_{\hat{\mu} s}^{\alpha a}=\frac{i}{N} \sum_{i} \hat{\mu}_{i}^{(\alpha)} s_{i}^{(a)}$ \\
$C_{\hat{h} \hat{h}}=-\frac{1}{N} \sum_{i} \hat{h}_{i} \hat{h}_{i}$ & $C_{\hat{h} \hat{\eta}}^{1 \alpha}=-\frac{1}{N} \sum_{i} \hat{h}_{i} \hat{\eta}_{i}^{(\alpha)}$ & $C_{\hat{h} \hat{\mu}}^{1 \alpha}=-\frac{1}{N} \sum_{i} \hat{h}_{i} \hat{\mu}_{i}^{(\alpha)}$ \\
$m_{\sigma}=\frac{1}{N} \sum_{i} \sigma_{i}^{(\alpha)}$ & $Q_{s s \sigma}^{1 a \alpha}=\frac{1}{N} \sum_{i} s_{i} s_{i}^{a} \sigma_{i}^{\alpha}$ & \\
\hline
\end{tabular}

the conjugate order parameters we get

$$
\begin{aligned}
& \mathbb{E}_{J}\left[\mathcal{N}_{n m}\right]=\int \mathcal{D} \mathcal{B \mathcal { B }} \exp \left\{i \sum_{i} \hat{h}_{i} h_{i}+i \sum_{\alpha, i} \hat{\eta}_{i}^{(\alpha)} \eta_{i}^{(\alpha)}+i \sum_{\alpha, i} \hat{\mu}_{i}^{(\alpha)} \mu_{i}^{(\alpha)}\right\} \prod_{\alpha \leq \beta} \frac{d \hat{Q}_{\hat{\eta} \hat{\eta}}^{\alpha \beta} d Q_{\hat{\eta} \hat{\eta}}^{\alpha \beta}}{2 \pi} \\
& \prod_{\alpha \leq \beta} \frac{d \hat{Q}_{\hat{\mu} \hat{\mu}}^{\alpha \beta} d Q_{\hat{\mu} \hat{\mu}}^{\alpha \beta}}{2 \pi} \prod_{\alpha \beta} \frac{d \hat{Q}_{\hat{\eta} \hat{\mu}}^{\alpha \beta} d Q_{\hat{\eta} \hat{\mu}}^{\alpha \beta}}{2 \pi} \prod_{\alpha} \frac{d \hat{C}_{\hat{h} s \sigma}^{11 \alpha} d C_{\hat{h} s \sigma}^{11 \alpha}}{2 \pi} \prod_{\alpha \beta} \frac{d \hat{Q}_{\hat{\eta} s \sigma}^{\alpha 1 \beta} d Q_{\hat{\eta} s \sigma}^{\alpha 1 \beta}}{2 \pi} \\
& \prod_{\alpha \beta} \frac{d \hat{Q}_{\hat{\mu} s \sigma}^{\alpha 1 \beta} d Q_{\hat{\mu} s \sigma}^{\alpha 1 \beta}}{2 \pi} \prod_{a} \frac{d \hat{Q}_{\hat{h} s}^{1 a} d Q_{\hat{h} s}^{1 a}}{2 \pi} \prod_{a \alpha} \frac{d \hat{Q}_{\hat{\eta} s}^{\alpha a} d Q_{\hat{\eta} s}^{\alpha a}}{2 \pi} \prod_{a \alpha} \frac{d \hat{Q}_{\hat{\mu} s}^{\alpha a} d Q_{\hat{\mu} s}^{\alpha a}}{2 \pi} \\
& \prod_{\alpha<\beta} \frac{d \hat{Q}_{\sigma \sigma}^{\alpha \beta} d Q_{\sigma \sigma}^{\alpha \beta}}{2 \pi} \prod_{a<b} \frac{d \hat{Q}_{s s}^{a b} d Q_{s s}^{a b}}{2 \pi} \prod_{\alpha} \frac{d \hat{m}_{\sigma}^{\alpha} d m_{\sigma}^{\alpha}}{2 \pi} \prod_{a \neq 1 \alpha} \frac{d \hat{Q}_{s s \sigma}^{1 a \alpha} d Q_{s s \sigma}^{1 a \alpha}}{2 \pi} \\
& \frac{d \hat{C}_{\hat{h} \hat{h}} d C_{\hat{h} \hat{h}}}{2 \pi} \prod_{\alpha} \frac{d \hat{C}_{\hat{h} \hat{\eta}}^{1 \alpha} d C_{\hat{h} \hat{\eta}}^{1 \alpha}}{2 \pi} \prod_{\alpha} \frac{d \hat{C}_{\hat{h} \hat{\mu}}^{1 \alpha} d C_{\hat{h} \hat{\mu}}^{1 \alpha}}{2 \pi} \\
& \exp [N(-\Psi(\{Q, \hat{Q}\})+\Omega(\{Q\}))] \\
& \sum_{\{\underline{s}\}} \sum_{\{\underline{\sigma}\}} \prod_{\alpha=1}^{m} \mathbb{I}_{\underline{\sigma}^{(\alpha)}}^{r}\left(\underline{s}^{(1)}\right) \prod_{i} \exp \left[N \Phi_{\left\{s_{i}\right\},\left\{\sigma_{i}\right\}}(\hat{\mathcal{B}},\{\hat{Q}\})\right]
\end{aligned}
$$

where the auxiliary function $\Psi(\{Q, \hat{Q}\}), \Omega(\{Q\}))$ and $\Phi_{\left\{s_{i}\right\},\left\{\sigma_{i}\right\}}(\hat{\mathcal{B}},\{\hat{Q}\})$ are defined by:

$$
\begin{aligned}
-i \Psi(\{Q, \hat{Q}\})= & {\left[\sum_{\alpha \leq \beta} \hat{Q}_{\hat{\eta} \hat{\eta}}^{\alpha \beta} Q_{\hat{\eta} \hat{\eta}}^{\alpha \beta}+\sum_{\alpha \leq \beta} \hat{Q}_{\hat{\mu} \hat{\mu}}^{\alpha \beta} Q_{\hat{\mu} \hat{\mu}}^{\alpha \beta}+\sum_{\alpha \beta} \hat{Q}_{\hat{\eta} \hat{\mu}}^{\alpha \beta} Q_{\hat{\eta} \hat{\mu}}^{\alpha \beta}+\sum_{\alpha<\beta} \hat{Q}_{\sigma \sigma}^{\alpha \beta} Q_{\sigma \sigma}^{\alpha \beta}+\right.} \\
& +\sum_{a<b} \hat{Q}_{s s}^{a b} Q_{s s}^{a b}+\sum_{\alpha} \hat{C}_{\hat{h} \hat{\eta}}^{1 \alpha} C_{\hat{h} \hat{\eta}}^{1 \alpha}+\sum_{\alpha} \hat{C}_{\hat{h} \hat{\mu}}^{1 \alpha} C_{\hat{h} \hat{\mu}}^{1 \alpha}+\sum_{\alpha} \hat{m}_{\sigma}^{\alpha} m_{\sigma}^{\alpha}+ \\
& +\sum_{\alpha} \hat{C}_{\hat{h} s \sigma}^{11 \alpha} C_{\hat{h} s \sigma}^{11 \alpha}+\sum_{\alpha \beta} \hat{Q}_{\hat{\eta} s \sigma}^{\alpha 1 \beta} Q_{\hat{\eta} s \sigma}^{\alpha 1 \beta}+\sum_{\alpha \beta} \hat{Q}_{\hat{\mu} s \sigma}^{\alpha 1 \beta} Q_{\hat{\mu} s \sigma}^{\alpha 1 \beta}+\hat{C}_{\hat{h} \hat{h}} C_{\hat{h} \hat{h}}+ \\
& \left.+\sum_{a} \hat{Q}_{\hat{h} s}^{1 a} Q_{\hat{h} s}^{1 a}+\sum_{a \alpha} \hat{Q}_{\hat{\eta} s}^{\alpha a} Q_{\hat{\eta} s}^{\alpha a}+\sum_{a \alpha} \hat{Q}_{\hat{\mu} s}^{\alpha a} Q_{\hat{\mu} s}^{\alpha a}+\sum_{a \neq 1 \alpha} \hat{Q}_{s s \sigma}^{1 a \alpha} Q_{s s \sigma}^{1 a \alpha}\right]
\end{aligned}
$$




$$
\begin{aligned}
& \Omega(\{Q\})=3 C_{\hat{h} \hat{h}}+6\left(Q_{\hat{h} s}^{11}\right)^{2}+\sum_{\alpha \beta}\left(3 Q_{\hat{\eta} \hat{\eta}}^{\alpha \beta}\left(Q_{\sigma \sigma}^{\alpha \beta}\right)^{2}+6\left(Q_{\hat{\eta} s \sigma}^{\alpha 1 \beta}\right)^{2} Q_{\sigma \sigma}^{\alpha \beta}\right)+ \\
& +\sum_{\alpha \beta}\left(3 Q_{\hat{\mu} \hat{\mu}}^{\alpha \beta}\left(2 Q_{\sigma \sigma}^{\alpha \beta}+2 m_{\sigma}^{\alpha} m_{\sigma}^{\beta}\right)+6\left(Q_{\hat{\mu} s \sigma}^{\alpha 1 \beta}\right)^{2}+12 Q_{\hat{\mu} s}^{\alpha 1} Q_{\hat{\mu} s \sigma}^{\beta 1 \alpha} m_{\sigma}^{\beta}+6 Q_{\hat{\mu} s}^{\alpha 1} Q_{\hat{\mu} s}^{\beta 1} Q_{\sigma \sigma}^{\alpha \beta}\right)+ \\
& +\sum_{\alpha}\left(6 C_{\hat{h} \hat{\eta}}^{1 \alpha}\left(m_{\sigma}^{\alpha}\right)^{2}+12 C_{\hat{h} s \sigma}^{11 \alpha} Q_{\hat{\eta} s}^{\alpha 1} m_{\sigma}^{\alpha}+12 C_{\hat{h} \hat{\mu}}^{1 \alpha} m_{\sigma}^{\alpha}+12 C_{\hat{h} s \sigma}^{11 \alpha} Q_{\hat{\mu} s}^{\alpha 1}+12 Q_{\hat{h} s}^{11} Q_{\hat{\mu} s}^{\alpha 1} m_{\sigma}^{\alpha}\right)+ \\
& +\sum_{\alpha \beta}\left(12 Q_{\hat{\eta} \hat{\mu}}^{\alpha \beta} Q_{\sigma \sigma}^{\alpha \beta} m_{\sigma}^{\alpha}+12 Q_{\hat{\eta} s \sigma}^{\alpha 1 \beta} Q_{\hat{\mu} s \sigma}^{\alpha 1 \beta} m_{\sigma}^{\alpha}+12 Q_{\hat{\eta} s}^{\alpha 1} Q_{\hat{\mu} s \sigma}^{\alpha 1 \beta} Q_{\sigma \sigma}^{\alpha \beta}\right)+ \\
& +\frac{1}{4}\left(\sum_{a b}\left(Q_{s s}^{a b}\right)^{3} \beta^{2}+2 \beta\left(3 Q_{\hat{s} s}^{11}+3 \sum_{a \neq 1} Q_{\hat{h} s}^{1 a}\left(Q_{s s}^{a b}\right)^{2}+3 \sum_{\alpha} Q_{\hat{\eta} s}^{\alpha 1}\left(m_{\sigma}^{\alpha}\right)^{2}+\right.\right. \\
& \left.\left.+3 \sum_{\alpha} \sum_{a \neq 1} Q_{\hat{\eta} s}^{\alpha a}\left(Q_{s s \sigma}^{1 a \alpha}\right)^{2}+6 \sum_{\alpha} Q_{\hat{\mu} s}^{\alpha 1} m_{\sigma}^{\alpha}+6 \sum_{\alpha} \sum_{a \neq 1} Q_{\hat{\mu} s}^{\alpha a} Q_{s s \sigma}^{1 a \alpha} Q_{s s}^{1 a}\right)\right), \\
& i \Phi_{\left\{s_{i}\right\},\left\{\sigma_{i}\right\}}(\hat{\mathcal{B}},\{\hat{Q}\})=\left[\sum_{\alpha \leq \beta} \hat{Q}_{\hat{\eta} \hat{\eta}}^{\alpha \beta} \hat{\eta}_{i}^{(\alpha)} \hat{\eta}_{i}^{(\beta)}+\sum_{\alpha \leq \beta} \hat{Q}_{\hat{\mu} \hat{\mu}}^{\alpha \beta} \hat{\mu}_{i}^{(\alpha)} \hat{\mu}_{i}^{(\beta)}+\sum_{\alpha \beta} \hat{Q}_{\hat{\eta} \hat{\mu}}^{\alpha \beta} \hat{\eta}_{i}^{(\alpha)} \hat{\mu}_{i}^{(\beta)}\right. \\
& -\sum_{\alpha<\beta} \hat{Q}_{\sigma \sigma}^{\alpha \beta} \sigma_{i}^{(\alpha)} \sigma_{i}^{(\beta)}-\sum_{a<b} \hat{Q}_{s s}^{a b} s_{i}^{(a)} s_{i}^{(b)}+\sum_{\alpha} \hat{C}_{\hat{h} \hat{\eta}}^{1 \alpha} \hat{h}_{i} \hat{\eta}_{i}^{(\alpha)}+\sum_{\alpha} \hat{C}_{\hat{h} \hat{\mu}}^{1 \alpha} \hat{h}_{i} \hat{\mu}_{i}^{(\alpha)} \\
& -\sum_{\alpha} \hat{m}_{\sigma}^{\alpha} \sigma_{i}^{(\alpha)}-i \sum_{a} \hat{Q}_{\hat{h} s}^{1 a} \hat{h}_{i} s_{i}^{(a)}-i \sum_{a \alpha} \hat{Q}_{\hat{\eta} s}^{\alpha a} \hat{\eta}_{i}^{(\alpha)} s_{i}^{(a)}-i \sum_{a \alpha} \hat{Q}_{\hat{\mu} s}^{\alpha a} \hat{\mu}_{i}^{(\alpha)} s_{i}^{(a)} \\
& -\sum_{a \neq 1 \alpha} \hat{Q}_{s s \sigma}^{1 a \alpha} s_{i}^{(1)} s_{i}^{(a)} \sigma_{i}^{(\alpha)}+\hat{C}_{\hat{h} \hat{h}} \hat{h}_{i} \hat{h}_{i}-i \sum_{\alpha \beta} \hat{Q}_{\hat{\eta} s \sigma}^{\alpha 1 \beta} \hat{\eta}_{i}^{(\alpha)} s_{i}^{(1)} \sigma_{i}^{(\beta)} \\
& \left.-i \sum_{\alpha \beta} \hat{Q}_{\hat{\mu} s \sigma}^{\alpha 1 \beta} \hat{\mu}_{i}^{(\alpha)} s_{i}^{(1)} \sigma_{i}^{(\beta)}-i \sum_{\alpha} \hat{C}_{\hat{h} s \sigma}^{11 \alpha} \hat{h}_{i} s_{i}^{(1)} \sigma_{i}^{(\alpha)}\right] .
\end{aligned}
$$

At this point we adopt the RS ansatz illustrated in Table II and Table III. As mentioned in the Main Text, one should invoke a more complex hierarchical ansatz [3] when averaging over configurations $\underline{s}$ but the RS ansatz is valid for all temperatures higher than $T_{K}$ [35]. In fact, in this regime, the paramagnetic state is replaced by an exponential number of states whose overlap is zero [36-38], from which a trivial Parisi function $P(q)=\delta(q)$ is obtained. Our results are thus valid as long as $T>T_{K}$. We also employ an RS ansatz for the $\sigma$-related order parameters; since $\sigma$ variables are just labels used to define clusters, there is no obvious reason why a more complicated scheme should be invoked. Let us notice that, by definition, $Q_{s s}^{a a}=Q_{\sigma \sigma}^{\alpha \alpha}=1$ and thus thus we do not have the conjugate order parameters $\hat{Q}_{s s}^{a a}$ and $\hat{Q}_{\sigma \sigma}^{\alpha \alpha}$. Similarly, since $Q_{s s \sigma}^{11 \alpha}=m_{\sigma}^{\alpha}$ we do not have to define nor $Q_{s s \sigma}^{11 \alpha}$ nor $\hat{Q}_{s s \sigma}^{11 \alpha}$. Moreover, we can 


\section{TABLE II.}

\begin{tabular}{c}
\hline \multicolumn{1}{c}{$\forall \alpha$} \\
\hline$\hat{m}_{\sigma}^{\alpha}=\hat{m}_{\sigma} \quad m_{\sigma}^{\alpha}=m_{\sigma}$ \\
$\hat{C}_{\hat{h} \hat{\eta}}^{1 \alpha}=\hat{C}_{\hat{h} \hat{\eta}} \quad C_{\hat{h} \hat{\eta}}^{1 \alpha}=C_{\hat{h} \hat{\eta}}$ \\
$\hat{C}_{\hat{h} \hat{\mu}}^{1 \alpha}=\hat{C}_{\hat{h} \hat{\mu}} \quad C_{\hat{h} \hat{\mu}}^{1 \alpha}=C_{\hat{h} \hat{\mu}}$ \\
$\hat{C}_{\hat{h} s \sigma}^{11 \alpha}=\hat{C}_{\hat{h} s \sigma} C_{\hat{h} s \sigma}^{11 \alpha}=C_{\hat{h} s \sigma}$ \\
\hline
\end{tabular}

TABLE III.

\begin{tabular}{|c|c|c|}
\hline \multicolumn{2}{|c|}{$\alpha \neq \beta$} & $\alpha=\beta$ \\
\hline$\hat{Q}_{\hat{\eta} \hat{\eta}}^{\alpha \beta}=\hat{Q}_{\hat{\eta} r}$ & $Q_{\hat{\eta} \hat{\eta}}^{\alpha \beta}=Q_{\hat{\eta} \hat{\eta}}$ & $\hat{Q}_{\hat{\eta} \hat{\eta}}^{\alpha \beta}=\hat{C}_{\hat{\eta} \hat{\eta}} \quad Q_{\hat{\eta} \hat{\eta}}^{\alpha \beta}=C_{\hat{\eta} \hat{\eta}}$ \\
\hline$\hat{Q}_{\hat{\mu} \hat{\mu}}^{\alpha \beta}=\hat{Q}_{\hat{\mu}}$ & $Q_{\hat{\mu} \hat{\mu}}^{\alpha \beta}=Q_{\hat{\mu} \hat{\mu}}$ & $\hat{Q}_{\hat{\mu} \hat{\mu}}^{\alpha \beta}=\hat{C}_{\hat{\mu} \hat{\mu}} \quad Q_{\hat{\mu} \hat{\mu}}^{\alpha \beta}=C_{\hat{\mu} \hat{\mu}}$ \\
\hline$\hat{Q}_{\hat{\eta} \hat{\mu}}^{\alpha \beta}=\hat{Q}_{\hat{\eta} t}$ & $Q_{\hat{\eta} \hat{\mu}}^{\alpha \beta}=Q_{\hat{\eta} \hat{\mu}}$ & $\hat{Q}_{\hat{\eta} \hat{\mu}}^{\alpha \beta}=\hat{C}_{\hat{\eta} \hat{\mu}} \quad Q_{\hat{\eta} \hat{\mu}}^{\alpha \beta}=C_{\hat{\eta} \hat{\mu}}$ \\
\hline$\hat{Q}_{\sigma \sigma}^{\alpha \beta}=\hat{Q}_{\sigma c}$ & $Q_{\sigma \sigma}^{\alpha \beta}=Q_{\sigma \sigma}$ & \\
\hline \multirow{2}{*}{\multicolumn{3}{|c|}{$\begin{array}{l}\hat{Q}_{\hat{\eta} s \sigma}^{\alpha 1 \beta}=\hat{Q}_{\hat{\eta} s \sigma} Q_{\hat{\eta} s \sigma}^{\alpha 1 \beta}=Q_{\hat{\eta} s \sigma} \hat{Q}_{\hat{\eta} s \sigma}^{\alpha 1 \beta}=\hat{C}_{\hat{\eta} s \sigma} Q_{\hat{\eta} s \sigma}^{\alpha 1 \beta}=C_{\hat{\eta} s \sigma} \\
\hat{Q}_{\hat{\mu} s \sigma}^{\alpha 1 \beta}=\hat{Q}_{\hat{\mu} s \sigma} Q_{\hat{\mu} s \sigma}^{\alpha 1 \beta}=Q_{\hat{\mu} s \sigma} \hat{Q}_{\hat{\mu} s \sigma}^{\alpha 1 \beta}=\hat{C}_{\hat{\mu} s \sigma} Q_{\hat{\mu} s \sigma}^{\alpha 1 \beta}=C_{\hat{\mu} s \sigma}\end{array}$}} \\
\hline & & \\
\hline \multicolumn{2}{|c|}{$a \neq 1$} & $a=1$ \\
\hline$\hat{Q}_{\hat{h} s}^{1 a}=\hat{Q}_{\hat{h} s}$ & $Q_{\hat{h} s}^{1 a}=Q_{\hat{h} s}$ & $\hat{Q}_{\hat{h} s}^{1 a}=\hat{C}_{\hat{h} s}$ \\
\hline$\hat{Q}_{\hat{\eta} s}^{\alpha a}=\hat{Q}_{\hat{\eta} s}$ & $Q_{\hat{\eta} s}^{\alpha a}=Q_{\hat{\eta} s}$ & $\hat{Q}_{\hat{\eta} s}^{\alpha a}=\hat{C}_{\hat{\eta} s}$ \\
\hline$\hat{Q}_{\hat{\mu} s}^{\alpha a}=\hat{Q}_{\hat{\mu} s}$ & $Q_{\hat{\mu} s}^{\alpha a}=Q_{\hat{\mu} s}$ & $\hat{Q}_{\hat{\mu} s}^{\alpha a}=\hat{C}_{\hat{\mu} s} \quad Q_{\hat{\mu} s}^{\alpha a}=C_{\hat{\mu} s}$ \\
\hline \multicolumn{3}{|c|}{$\hat{Q}_{s s \sigma}^{1 a \alpha}=\hat{Q}_{s s \sigma} \quad Q_{s s \sigma}^{1 a \alpha}=Q_{s s \sigma}$} \\
\hline \multicolumn{3}{|c|}{$a \neq b$} \\
\hline$\hat{Q}_{s s}^{a b}=\hat{Q}_{s s}$ & $Q_{s s}^{a b}=Q_{s s}$ & \\
\hline
\end{tabular}

perform the integration over $m_{\sigma}$, which fixes $m_{\sigma}$ to be equal to $2 r-1$, thanks to the delta functions contained in the Eq. (54). Thanks to the RS ansatz, we can easily linearize the quadratic terms in eq. (59) in order to compute the sums over $\{\underline{\sigma}\},\{\underline{\sigma}\}$ and the integrals over $\underline{\hat{h}},\{\hat{\eta}\}$ and $\{\hat{\mu}\}$. The linearization can be done thanks to the Hubbard-Stratonovich transformation, which introduces the integration variables $\underline{z}$ and $\underline{x}$, as can be seen in eq. (69) 
and eq. (71). These integrals result in three delta functions, which lead to the expressions

$$
\begin{aligned}
& h_{i}=z_{1}+s_{i}^{(1)} \Delta_{\hat{h} s}, \\
& \eta_{i}^{(\alpha)}=z_{2}+x_{1}+s_{i}^{(1)} \Delta_{\hat{\eta} s}+s_{i}^{(1)} \sigma_{i}^{(\alpha)} \Delta_{\hat{\eta} s \sigma}, \\
& \mu_{i}^{(\alpha)}=z_{3}+x_{2}+s_{i}^{(1)} \Delta_{\hat{\mu} s}+s_{i}^{(1)} \sigma_{i}^{(\alpha)} \Delta_{\hat{\mu} s \sigma},
\end{aligned}
$$

for the three fields contained in the Heaviside function in eq. (54). According to the value of the spin $s_{i}^{(1)}$ on which we have to sum over, we define the quantity

$$
\theta_{\underline{x}, \underline{z}}^{ \pm}(\{\Delta\})=\theta\left[\left(z_{1} \pm \Delta_{\hat{h} s}\right)\left(z_{2}+x_{1} \pm \Delta_{\hat{\eta} s} \pm \Delta_{\hat{\eta} s \sigma}+z_{3}+x_{2} \pm \Delta_{\hat{\mu} s} \pm \Delta_{\hat{\mu} s \sigma}-z_{1} \mp \Delta_{\hat{h} s}\right)\right]
$$

where we defined

$$
\{\Delta\}=\left\{\begin{array}{l}
\Delta_{\hat{h} s}=\hat{C}_{\hat{h} s}-\hat{Q}_{\hat{h} s} \\
\Delta_{\hat{\eta} s}=\hat{C}_{\hat{\eta} s}-\hat{Q}_{\hat{\eta} s} . \\
\Delta_{\hat{\mu} s}=\hat{C}_{\hat{\mu} s}-\hat{Q}_{\hat{\mu} s}
\end{array} .\right.
$$

The RS expression of the quantity given in eq. (53) is thus

$$
\mathbb{E}_{J}\left[\mathcal{N}_{n m}\right]=\int \mathcal{D} \mathcal{Q} \hat{\mathcal{Q}} \exp [N(-\Psi(\{Q, \hat{Q}\})+\Omega(\{Q\}))+\Phi(\{\hat{Q}\})]
$$

where the integration measure $\mathcal{D} \mathcal{Q} \hat{\mathcal{Q}}$ contains all the order parameters defined in Table II and in Table III, except $m_{\sigma}$. The three function in the exponent come, respectively, from the integral representation of the order parameters, from the average over disorder and from the sum over the spins. Their expressions are

$$
\begin{aligned}
\Psi(\{Q, \hat{Q}\})= & \frac{m(m-1)}{2} \hat{Q}_{\hat{\eta} \hat{\eta}} Q_{\hat{\eta} \hat{\eta}}+m \hat{C}_{\hat{\eta} \hat{\eta}} C_{\hat{\eta} \hat{\eta}}+\frac{m(m-1)}{2} \hat{Q}_{\hat{\mu} \hat{\mu}} Q_{\hat{\mu} \hat{\mu}}+m \hat{C}_{\hat{\mu} \hat{\mu}} C_{\hat{\mu} \hat{\mu}}+ \\
& +m(m-1) \hat{Q}_{\hat{\eta} \hat{\mu}} Q_{\hat{\eta} \hat{\mu}}+m \hat{C}_{\hat{\eta} \hat{\mu}} C_{\hat{\eta} \hat{\mu}}+\frac{m(m-1)}{2} \hat{Q}_{\sigma \sigma} Q_{\sigma \sigma}+\frac{n(n-1)}{2} \hat{Q}_{s s} Q_{s s}+ \\
& +m \hat{C}_{\hat{h} \hat{\eta}} C_{\hat{h} \hat{\eta}}+m \hat{C}_{\hat{h} \hat{\mu}} C_{\hat{h} \hat{\mu}}+m \hat{m}_{\sigma} m_{\sigma}+m \hat{C}_{\hat{h} s \sigma} C_{\hat{h} s \sigma}+\hat{C}_{\hat{h} \hat{h}} C_{\hat{h} \hat{h}}+ \\
& +m(m-1) \hat{Q}_{\hat{\eta} s \sigma} Q_{\hat{\eta} s \sigma}+m \hat{C}_{\hat{\eta} s \sigma} C_{\hat{\eta} s \sigma}+m(m-1) \hat{Q}_{\hat{\mu} s \sigma} Q_{\hat{\mu} s \sigma}+m \hat{C}_{\hat{\mu} s \sigma} C_{\hat{\mu} s \sigma}+ \\
& +\hat{C}_{\hat{h} s} C_{\hat{h} s}+(n-1) \hat{Q}_{\hat{h} s} Q_{\hat{h} s}+m \hat{C}_{\hat{\eta} s} C_{\hat{\eta} s}+m(n-1) \hat{Q}_{\hat{\eta} s} Q_{\hat{\eta} s}+ \\
& +m \hat{C}_{\hat{\mu} s} C_{\hat{\mu} s}+m(n-1) \hat{Q}_{\hat{\mu} s} Q_{\hat{\mu} s}+m(n-1) \hat{Q}_{s s \sigma} Q_{s s \sigma}+ \\
& +\frac{n}{2} \hat{Q}_{s s}+\frac{m}{2} \hat{Q}_{\sigma \sigma}+m \hat{Q}_{s s \sigma} m_{\sigma},
\end{aligned}
$$




$$
\begin{aligned}
& \Omega(\{Q\})= 3 C_{\hat{h} \hat{h}}+6 C_{\hat{h} s}^{2}+3 m(m-1)\left[Q_{\hat{\eta} \hat{\eta}} Q_{\sigma \sigma}^{2}+2 Q_{\hat{\eta} s \sigma}^{2} Q_{\sigma \sigma}\right]+3 m\left[C_{\hat{\eta} \hat{\eta}}+2 C_{\hat{\eta} s \sigma}^{2}\right]+ \\
&+6 m(m-1)\left[Q_{\hat{\mu} \hat{\mu}} Q_{\sigma \sigma}+Q_{\hat{\mu} \hat{\mu}} m_{\sigma}^{2}\right]+6 m\left[C_{\hat{\mu} \hat{\mu}}+C_{\hat{\mu} \hat{\mu}} m_{\sigma}^{2}\right]+6 m(m-1) Q_{\hat{\mu} s \sigma}^{2}+ \\
&+6 m C_{\hat{\mu} s \sigma}^{2}+12 m(m-1) C_{\hat{\mu} s} Q_{\hat{\mu} s \sigma} m_{\sigma}+12 m C_{\hat{\mu} s} C_{\hat{\mu} s \sigma} m_{\sigma}+6 m(m-1) C_{\hat{\mu} s}^{2} Q_{\sigma \sigma}+ \\
&+6 m C_{\hat{\mu} s}^{2}+6 m C_{\hat{h} \hat{\eta}} m_{\sigma}^{2}+12 m C_{\hat{h} s \sigma} C_{\hat{\eta} s} m_{\sigma}+12 m C_{\hat{h} \hat{\mu}} m_{\sigma}+12 m C_{\hat{h} s \sigma} C_{\hat{\mu} s}+ \\
&+12 m C_{\hat{h} s} C_{\hat{\mu} s} m_{\sigma}+12 m(m-1) Q_{\hat{\eta} \hat{\eta}} Q_{\sigma \sigma} m_{\sigma}+12 m C_{\hat{\eta} \hat{\mu}} m_{\sigma}+12 m C_{\hat{\eta} s \sigma} C_{\hat{\mu} s \sigma} m_{\sigma}+ \\
&+12 m(m-1) Q_{\hat{\eta} s \sigma} Q_{\hat{\mu} s \sigma} m_{\sigma}+12 m(m-1) C_{\hat{\eta} s} Q_{\hat{\mu} s \sigma} Q_{\sigma \sigma}+12 m C_{\hat{\eta} s} C_{\hat{\mu} s \sigma}+ \\
&+\frac{1}{4}\left[n(n-1) \beta^{2} Q_{s s}^{3}+\beta^{2} n+2 \beta\left(3 C_{\hat{h} s}+3(n-1) Q_{\hat{h} s} Q_{s s}^{2}+3 m C_{\hat{\eta} s} m_{\sigma}^{2}+\right.\right. \\
&\left.\left.+3 m(n-1) Q_{\hat{\eta} s} Q_{s s \sigma}^{2}+6 m C_{\hat{\mu} s} m_{\sigma}+6 m(n-1) Q_{\hat{\mu} s} Q_{s s \sigma} Q_{s s}\right)\right] \\
& \Phi(\{\hat{Q}\})=\log \left[\int D \underline{z}^{+} e^{z_{5}-\left(z_{4}+\hat{m}_{\sigma}\right) m}\left(1+m g^{+}\left(\hat{m}_{\sigma},\{\Delta\}\right)\right)\left(2 \cosh z_{5}\right)^{n-1}+\right. \\
&\left.+\int D \underline{z}^{-} e^{-z_{5}-\left(z_{4}+\hat{m}_{\sigma}\right) m}\left(1+m g^{-}\left(\hat{m}_{\sigma},\{\Delta\}\right)\right)\left(2 \cosh z_{5}\right)^{n-1}\right]
\end{aligned}
$$

and in the last expression, similarly to what has been done in eq. (23), we defined the measure

$$
\begin{aligned}
D \underline{z}^{ \pm} & =\sqrt{\frac{\operatorname{det}\left(U^{ \pm}\right)^{-1}}{(2 \pi)^{5}}} \prod_{k=1}^{5} d z_{k} \exp \left\{-\frac{1}{2} \underline{z}^{T}\left(U^{ \pm}\right)^{-1} \underline{z}\right\}, \\
U^{ \pm}= & \left(\begin{array}{ccccc}
2 \hat{C}_{\hat{h} \hat{h}} & \hat{C}_{\hat{h} \hat{\eta}} & \hat{C}_{\hat{h} \hat{\mu}} & \pm \hat{C}_{\hat{h} s \sigma} & \hat{Q}_{\hat{h} s} \\
\hat{C}_{\hat{h} \hat{\eta}} & \hat{Q}_{\hat{\eta} \hat{\eta}} & \hat{Q}_{\hat{\eta} \hat{\mu}} & \pm \hat{Q}_{\hat{\eta} s \sigma} & \hat{Q}_{\hat{\eta} s} \\
\hat{C}_{\hat{h} \hat{\mu}} & \hat{Q}_{\hat{\eta} \hat{\mu}} & \hat{Q}_{\hat{\mu} \hat{\mu}} & \pm \hat{Q}_{\hat{\mu} s \sigma} & \hat{Q}_{\hat{\mu} s} \\
\pm \hat{C}_{\hat{h} s \sigma} & \pm \hat{Q}_{\hat{\eta} s \sigma} & \pm \hat{Q}_{\hat{\mu} s \sigma} & \hat{Q}_{\sigma \sigma} & \pm \hat{Q}_{s s \sigma} \\
\hat{Q}_{\hat{h} s} & \hat{Q}_{\hat{\eta} s} & \hat{Q}_{\hat{\mu} s} & \pm \hat{Q}_{s s \sigma} & \hat{Q}_{s s}
\end{array}\right),
\end{aligned}
$$

and the function

$$
g^{ \pm}\left(\hat{m}_{\sigma},\{\Delta\}\right)=\log \left[1+e^{2\left(z_{4}+\hat{m}_{\sigma}\right)} \int D \underline{x} \theta_{\underline{x}, \underline{z}}^{ \pm}(\{\Delta\})\right]
$$

with the corresponding measure

$$
D \underline{x}=\sqrt{\frac{\operatorname{det} V^{-1}}{(2 \pi)^{2}}} \prod_{k=1}^{2} d x_{k} \exp \left\{-\frac{1}{2} \underline{x}^{T} V^{-1} \underline{x}\right\}, \quad V=\left(\begin{array}{cc}
\delta_{\hat{\eta} \hat{\eta}} & \Delta_{\hat{\eta} \hat{\mu}} \\
\Delta_{\hat{\eta} \hat{\mu}} & \delta_{\hat{\mu} \hat{\mu}}
\end{array}\right),
$$

where the entries of the matrix $V$ are given by

$$
\begin{aligned}
\delta_{\hat{\eta} \hat{\eta}} & =2 \hat{C}_{\hat{\eta} \hat{\eta}}-\hat{Q}_{\hat{\eta} \hat{\eta}} \\
\delta_{\hat{\mu} \hat{\mu}} & =2 \hat{C}_{\hat{\mu} \hat{\mu}}-\hat{Q}_{\hat{\mu} \hat{\mu}}, \\
\Delta_{\hat{\eta} \hat{\mu}} & =\hat{C}_{\hat{\eta} \hat{\mu}}-\hat{Q}_{\hat{\eta} \hat{\mu}}
\end{aligned}
$$


and the function $\theta_{\underline{x}, \underline{z}}^{ \pm}(\{\Delta\})$ has been defined in eq. (63). The integral over the inner measure in eq. (70) can be done analytically using a result that we will mention later, see equations (145)-(146), and it is equal to

$$
L_{ \pm}=\int D \underline{x} \theta_{\underline{x}, \underline{z}}^{ \pm}(\{\Delta\})=1-\frac{1}{2} \operatorname{erfc}\left[\frac{c_{ \pm}}{\sqrt{2 D a_{ \pm}^{2}}}\right]
$$

where $\operatorname{erfc}(x)$ is the complementary error function,

$$
\operatorname{erfc}(x)=1-\frac{2}{\sqrt{\pi}} \int_{0}^{x} e^{-t^{2}} d t
$$

and the parameters appearing in its argument are defined by

$$
\begin{gathered}
D=V_{11}+V_{22}+2 V_{12}, \\
a_{ \pm}=z_{1} \pm \Delta_{\hat{h} s}, \\
c_{ \pm}=\left[\left(z_{2} \pm \Delta_{\hat{\eta} s} \pm \Delta_{\hat{\eta} s \sigma}\right)+\left(z_{3} \pm \Delta_{\hat{\mu} s} \pm \Delta_{\hat{\mu} s \sigma}\right)-\left(z_{1} \pm \Delta_{\hat{h} s}\right)\right]\left(z_{1} \pm \Delta_{\hat{h} s}\right) .
\end{gathered}
$$

Having introduced all these definitions, we can evaluate the integral in eq. (65) with the steepest descent method, after $-i\{\hat{Q}\}=\{\hat{Q}\}$, where $\{Q\}$ is the set of all the order parameters involved. The saddle point equations obtained optimizing with respect to the original order parameters read

$$
\begin{aligned}
& \frac{n(n-1)}{2} \hat{Q}_{s s}= \frac{3}{4} \beta^{2}(n-1) m Q_{s s}^{2}+\frac{1}{4}\left(6 \beta(n-1) Q_{\hat{h} s} Q_{s s}+12 \beta m(n-1) Q_{\hat{\mu} s} Q_{s s \sigma}\right), \\
& \hat{Q}_{s s \sigma}= \frac{3}{2}\left[2 \beta Q_{\hat{\eta} s} Q_{s s \sigma}+Q_{\hat{\mu} s} Q_{s s}\right], \\
& \hat{Q}_{\sigma \sigma}= 3\left[Q_{\hat{\eta} \hat{\eta}} Q_{\sigma \sigma}+Q_{\hat{\eta} s \sigma}^{2}+Q_{\hat{\mu} \hat{\mu}}+C_{\hat{\mu} s}^{2}+2 Q_{\hat{\eta} \hat{\mu}} m_{\sigma}+2 C_{\hat{\eta} s} Q_{\hat{\mu} s \sigma}\right], \\
& \hat{C}_{\hat{h} s}= \frac{1}{4}\left(6 \beta+12 C_{\hat{h} s}+12 m C_{\hat{\mu} s} m_{\sigma}\right) \\
& \hat{C}_{\hat{\eta} s}= \frac{3}{2}\left(\beta m_{\sigma}^{2}+2 C_{\hat{h} s \sigma} m_{\sigma}+2(m-1) Q_{\hat{\mu} s \sigma} Q_{\sigma \sigma}+2 C_{\hat{\mu} s \sigma}\right) \\
& \hat{C}_{\hat{\mu} s}= \frac{3}{2}\left(\beta m_{\sigma}+2(m-1) Q_{\hat{\mu} s \sigma} m_{\sigma}+2 C_{\hat{\mu} s \sigma} m_{\sigma}+2(m-1) C_{\hat{\mu} s} Q_{\sigma \sigma}+\right. \\
&\left.+2 C_{\hat{\mu} s}+2 C_{\hat{h} s \sigma}+2 C_{\hat{h} s} m_{\sigma}\right) \\
& \hat{Q}_{\hat{h} s}= \frac{3}{2} \beta Q_{s s}^{2}, \\
& \hat{Q}_{\hat{\eta} s}=\frac{3}{2} \beta Q_{s s \sigma}^{2} \\
& \hat{Q}_{\hat{\mu} s}=3 \beta Q_{s s \sigma} Q_{s s},
\end{aligned}
$$




$$
\begin{gathered}
\hat{C}_{\hat{h} \hat{h}}=\frac{3}{4}, \\
\hat{C}_{\hat{\eta} \hat{\eta}}=\frac{3}{4}, \\
\hat{C}_{\hat{\mu} \hat{\mu}}=\frac{3}{2}\left(1+m_{\sigma}^{2}\right), \\
\hat{C}_{\hat{\eta} \hat{\mu}}=3 m_{\sigma}, \\
\hat{C}_{\hat{h} \hat{\eta}}=\frac{3}{2} m_{\sigma}^{2}, \\
\hat{C}_{\hat{h} \hat{\mu}}=3 m_{\sigma}, \\
\hat{Q}_{\hat{\eta} \hat{\eta}}=\frac{3}{2} Q_{\sigma \sigma}^{2}, \\
\hat{Q}_{\hat{\mu} \hat{\mu}}=3\left(Q_{\sigma \sigma}+m_{\sigma}^{2}\right), \\
\hat{Q}_{\hat{\eta} \hat{\mu}}=3 Q_{\sigma \sigma} m_{\sigma}, \\
\hat{C}_{\hat{h} s \sigma}=3\left[C_{\hat{\eta} s} m_{\sigma}+C_{\hat{\mu} s}\right] \\
\hat{C}_{\hat{\eta} s \sigma}=3\left[C_{\hat{\eta} s \sigma}+C_{\hat{\mu} s \sigma} m_{\sigma}\right], \\
\hat{C}_{\hat{\mu} s \sigma}=3\left[C_{\hat{\mu} s \sigma}+C_{\hat{\mu} s} m_{\sigma}+C_{\hat{\eta} s \sigma} m_{\sigma}+C_{\hat{\eta} s}\right] \\
\hat{Q}_{\hat{\eta} s \sigma}=3\left[Q_{\hat{\eta} s \sigma} Q_{\sigma \sigma}+Q_{\hat{\mu} s \sigma} m_{\sigma}\right], \\
\hat{Q}_{\hat{\mu} s \sigma}=3\left[Q_{\hat{\mu} s \sigma}+C_{\hat{\mu} s} m_{\sigma}+Q_{\hat{\eta} s \sigma} m_{\sigma}+C_{\hat{\eta} s} Q_{\sigma \sigma}\right] .
\end{gathered}
$$

In order to simplify the expressions of next saddle point equations we introduce the notation

$$
D_{i j}=\frac{\partial \Phi}{\partial U_{i j}^{+}}, \quad F_{i j}=\frac{\partial \Phi}{\partial V_{i j}} .
$$

At zero order in $m$ and $n$, it's easy to see that $D_{i j}^{0}=F_{i j}^{0}=0$. Let us also denote by

$$
\begin{aligned}
D_{i j}^{1, m} & \equiv \lim _{n \rightarrow 0} \lim _{m \rightarrow 0} \partial_{m} D_{i j}, \\
D_{i j}^{1, n} & \equiv \lim _{n \rightarrow 0} \lim _{m \rightarrow 0} \partial_{n} D_{i j} \\
F_{i j}^{1, m} & \equiv \lim _{n \rightarrow 0} \lim _{m \rightarrow 0} \partial_{m} F_{i j}
\end{aligned}
$$

their $O(m)$ and $O(n)$ contributions. Moreover, it is also useful to introduce

$$
G^{1, m} \equiv \lim _{n \rightarrow 0} \lim _{m \rightarrow 0} \frac{\partial}{\partial \hat{C}_{\bullet}} \partial_{m} \Phi,
$$

where we indicate by $\hat{C}_{\bullet}$ any of $\left\{\hat{C}_{\hat{\eta} s}, \hat{C}_{\hat{\mu} s}, \hat{C}_{\hat{\eta} s \sigma}, \hat{C}_{\hat{\mu} s \sigma}\right\}$, and

$$
M^{1, m} \equiv \lim _{n \rightarrow 0} \lim _{m \rightarrow 0} \frac{\partial}{\partial \hat{m}_{\sigma}} \partial_{m} \Phi .
$$


The saddle point equations obtained optimizing with respect to the conjugate order parameters thus read

$$
\begin{aligned}
& Q_{s s}=1-2 D_{55}^{1, n}, \\
& Q_{s s \sigma}=m_{\sigma}-D_{54}^{1, m}, \\
& Q_{\sigma \sigma}=1-2 D_{44}^{1, m}, \\
& C_{\hat{h} s}=O(m), \\
& C_{\hat{\eta} s}=G^{1, m}, \\
& C_{\hat{\mu} s}=G^{1, m}, \\
& Q_{\hat{h} s}=O(m) \text {, } \\
& Q_{\hat{\eta} s}=-D_{52}^{1, m}, \\
& Q_{\hat{\mu} s}=-D_{53}^{1, m} \text {, } \\
& C_{\hat{h} \hat{h}}=O(m), \\
& C_{\hat{\eta} \hat{\eta}}=2 F_{11}^{1, m}, \\
& C_{\hat{\mu} \hat{\mu}}=2 F_{22}^{1, m}, \\
& C_{\hat{\eta} \hat{\mu}}=2 F_{12}^{1, m}, \\
& C_{\hat{h} \hat{\eta}}=-D_{21}^{1, m} \text {, } \\
& C_{\hat{h} \hat{\mu}}=-D_{31}^{1, m} \text {, } \\
& Q_{\hat{\eta} \hat{\eta}}=-2 D_{22}^{1, m}, \\
& Q_{\hat{\mu} \hat{\mu}}=-2 D_{33}^{1, m}, \\
& Q_{\hat{\eta} \hat{\mu}}=-2 D_{23}^{1, m}, \\
& C_{\hat{h} s \sigma}=-D_{41}^{1, m}, \\
& C_{\hat{\eta} s \sigma}=G^{1, m}, \\
& C_{\hat{\mu} s \sigma}=G^{1, m} \text {, } \\
& Q_{\hat{\eta} s \sigma}=-D_{42}^{1, m} \text {, } \\
& Q_{\hat{\mu} s \sigma}=-D_{43}^{1, m},
\end{aligned}
$$




$$
m_{\sigma}=M^{1, m} .
$$

The reason why we have one equation more with respect to eq. (78)-(100) is that before we did not have to optimize over $m_{\sigma}$, while we still have to optimize over $\hat{m}_{\sigma}$. If we introduce the term

$$
x_{ \pm} \equiv \frac{c_{ \pm}}{\sqrt{2 D a_{ \pm}^{2}}},
$$

and the functions

$$
f^{ \pm}\left(z_{5}\right)=\frac{e^{ \pm z_{5}}}{2 \cosh \left(z_{5}\right)},
$$

the expressions for the matrices introduced above are

$$
\begin{aligned}
(i, j)= & \{(2,2),(3,3),(2,3)\}: \\
D_{i j}^{1, m}= & \int D \underline{z}^{+} T_{i j}^{+}(\underline{z})\left(-z_{4}+g^{+}\left(\hat{m}_{\sigma},\{\Delta\}\right)\right) f^{+}\left(z_{5}\right)+ \\
& \int D \underline{z}^{-} T_{i j}^{-}(\underline{z})\left(-z_{4}+g^{-}\left(\hat{m}_{\sigma},\{\Delta\}\right)\right) f^{-}\left(z_{5}\right)+ \\
& \int D \underline{z}^{+} f^{+}\left(z_{5}\right) \frac{e^{2\left(z_{4}+\hat{m}_{\sigma}\right)}}{e^{g_{+}}} \frac{e^{-x_{+}^{2}}}{\sqrt{\pi}} \frac{x_{+}}{2 D} \alpha+\int D \underline{z}^{-} f^{-}\left(z_{5}\right) \frac{e^{2\left(z_{4}+\hat{m}_{\sigma}\right)}}{e^{g_{-}}} \frac{e^{-x_{-}^{2}}}{\sqrt{\pi}} \frac{x_{-}}{2 D} \alpha, \\
& \text { where } \alpha=\left\{\begin{array}{l}
1(i, j)=\{(2,2),(3,3)\} \\
2(i, j)=\{(2,3)\}
\end{array}\right. \\
(i, j)= & \{(4,2),(4,3),(5,2),(5,3)\}: \\
D_{i j}^{1, m}= & \int D \underline{z}^{+} T_{i j}^{+}(\underline{z})\left(-z_{4}+g^{+}\left(\hat{m}_{\sigma},\{\Delta\}\right)\right) f^{+}\left(z_{5}\right)+ \\
& \alpha \int D \underline{z}^{-} T_{i j}^{-}(\underline{z})\left(-z_{4}+g^{-}\left(\hat{m}_{\sigma},\{\Delta\}\right)\right) f^{-}\left(z_{5}\right)+ \\
& -\int D \underline{z}^{+} f^{+}\left(z_{5}\right) \frac{e^{2\left(z_{4}+\hat{m}_{\sigma}\right)}}{e^{g_{+}}} \frac{e^{-x_{+}^{2}}}{\sqrt{\pi}} \frac{\operatorname{sgn}\left(a_{+}\right)}{\sqrt{2 D}}+\int D \underline{z}^{-} f^{-}\left(z_{5}\right) \frac{e^{2\left(z_{4}+\hat{m}_{\sigma}\right)}}{e^{g_{-}}} \frac{e^{-x_{-}^{2}}}{\sqrt{\pi}} \frac{\operatorname{sgn}\left(a_{-}\right)}{\sqrt{2 D}}, \\
& \text { where } \alpha=\left\{\begin{array}{l}
1(i, j)=\{(5,2),(5,3)\} \\
-1(i, j)=\{(4,2),(4,3)\}
\end{array},\right. \\
(i, j)= & \{(2,1),(3,1),(4,4)\}: \\
D_{i j}^{1, m}= & \int D \underline{z}^{+} T_{i j}^{+}(\underline{z})\left(-z_{4}+g^{+}\left(\hat{m}_{\sigma},\{\Delta\}\right)\right) f^{+}\left(z_{5}\right)+ \\
& \int D \underline{z}^{-} T_{i j}^{-}(\underline{z})\left(-z_{4}+g^{-}\left(\hat{m}_{\sigma},\{\Delta\}\right)\right) f^{-}\left(z_{5}\right),
\end{aligned}
$$




$$
\begin{aligned}
(i, j)= & \{(4,1),(5,4)\}: \\
D_{i j}^{1, m}= & \int D \underline{z}^{+} T_{i j}^{+}(\underline{z})\left(-z_{4}+g^{+}\left(\hat{m}_{\sigma},\{\Delta\}\right)\right) f^{+}\left(z_{5}\right)+ \\
& -\int D \underline{z}^{-} T_{i j}^{-}(\underline{z})\left(-z_{4}+g^{-}\left(\hat{m}_{\sigma},\{\Delta\}\right)\right) f^{-}\left(z_{5}\right),
\end{aligned}
$$

where the terms $T_{i j}^{ \pm}(\underline{z})$ are given by

$$
T_{i j}^{ \pm}(\underline{z})=\left\{\begin{array}{c}
\sum_{l p}\left[U^{ \pm}\right]_{l i}^{-1}\left[U^{ \pm}\right]_{p j}^{-1} z_{l} z_{p}-\left[U^{ \pm}\right]_{i j}^{-1} \quad i \neq j \\
\frac{1}{2} \sum_{l p}\left[U^{ \pm}\right]_{l i}^{-1}\left[U^{ \pm}\right]_{p i}^{-1} z_{l} z_{p}-\frac{1}{2}\left[U^{ \pm}\right]_{i i}^{-1} \quad i=j
\end{array}, \quad \underline{z}=\left\{z_{1}, z_{2}, z_{3}, z_{4}, z_{5}\right\}\right.
$$

and the matrix $U^{ \pm}$has been defined in eq. (69). Above we have the expressions of 12 terms, but the matrix $D_{i j}$, defined in eq. (101) should contain 15 independent entries. One of the missing terms is $D_{55}$ that we will describe shortly, while the other two are $D_{11}$ and $D_{51}$ which account for the $O(m)$ contributions in eq. (113) and eq. (116), that will be discussed later. $D_{55}$ appears in eq. (107) but we are interested in its $O(n)$ contribution rather than its $O(m)$ one:

$$
D_{55}^{1, n}=\frac{1}{2}-\frac{1}{2} \int D t \tanh ^{2}\left(t \sqrt{\hat{Q}_{s s}}\right) .
$$

We notice that with this expression, eq. (78) and eq. (107) correctly describe the reference system in the RS phase only under the hypothesis $Q_{\hat{h} s}=0, Q_{\hat{\mu} s} Q_{s s \sigma}=0$. In fact the reference system should not be affected by the order parameters related to the computation of the entropy of clusters, following the considerations made in the computation of the Franz-Parisi potential. Our approach to deal with this problem will be to assume $Q_{\hat{h} s}=0, Q_{s s \sigma}=0$ and check that these conditions hold self-consistently. Before discussing this problem, we give the expressions for the other matrix terms appearing in the equations above. The three terms $F_{i j}^{1 . m}$ appearing in equations (117)-(119) are given by

$$
\begin{aligned}
& F_{i j}^{1, m}=\int D \underline{z}^{+} f^{+}\left(z_{5}\right) \frac{\partial g^{+}}{\partial V_{i j}}+\int D \underline{z}^{-} f^{-}\left(z_{5}\right) \frac{\partial g^{-}}{\partial V_{i j}} \\
& \frac{\partial g^{ \pm}}{\partial V_{i j}}=\left\{e^{g^{ \pm}}\right\}^{-1}\left[e^{2\left(z_{4}+\hat{m}_{\sigma}\right)} \int D \underline{x} t_{i j}(\underline{x}) \theta_{\underline{x}, \underline{z}}^{ \pm}(\{\Delta\})\right]
\end{aligned}
$$


where

$$
t_{i j}(\underline{x})=\left\{\begin{array}{c}
\sum_{l p}[V]_{l i}^{-1}[V]_{p j}^{-1} x_{l} x_{p}-[V]_{i j}^{-1} i \neq j \\
\frac{1}{2} \sum_{l p}[V]_{l i}^{-1}[V]_{p i}^{-1} x_{l} x_{p}-\frac{1}{2}[V]_{i i}^{-1} i=j
\end{array} ; \quad \underline{x}=\left\{x_{1}, x_{2}\right\}\right.
$$

We introduced the term $G^{1, m}$ because differentiating $\Phi(\{\hat{Q}\})$ with respect to $\hat{C}_{\hat{\eta} s}, \hat{C}_{\hat{\mu} s}, \hat{C}_{\hat{\eta} s \sigma}$, $\hat{C}_{\hat{\mu} s \sigma}$ results in the same expression, as can be seen in eq. (63). This quantity is given by

$$
\begin{aligned}
G^{1, m}= & \int D \underline{z}^{+} f^{+}\left(z_{5}\right) i_{+}(\underline{z}) \frac{e^{2\left(\hat{m}_{\sigma}+z_{4}\right)}}{e^{g_{+}}}\left(z_{1}+\Delta_{\hat{h} s}\right)+ \\
& -\int D \underline{z}^{-} f^{-}\left(z_{5}\right) i_{-}(\underline{z}) \frac{e^{2\left(\hat{m}_{\sigma}+z_{4}\right)}}{e^{g_{-}}}\left(z_{1}-\Delta_{\hat{h}_{s}}\right),
\end{aligned}
$$

and it appears in eqs. (111), (112), (126) and (127). The integrals $i_{ \pm}(\underline{z})$, introduced above, can be defined by replacing the Heaviside function in eq. (73) by a Dirac delta,

$$
i_{ \pm}(\underline{z})=\int D \underline{x} \delta_{\underline{z}, \underline{x}}^{ \pm}(\{\Delta\})
$$

and are given by

$$
i_{ \pm}(\underline{z})=\frac{1}{\sqrt{2 \pi D}\left|a_{ \pm}\right|} \exp \left[-\frac{1}{2} D^{-1}\left(\frac{c_{ \pm}}{a_{ \pm}}\right)^{2}\right]
$$

and the parameters $a_{ \pm}, c_{ \pm}$have been defined in eqs. (76)-(77). Finally the term $M^{1, m}$, appearing in eq. (130), is given by

$$
M^{1, m}=-1+\int D \underline{z}^{+} f^{+}\left(z_{5}\right) \frac{\partial g^{+}}{\partial \hat{m}_{\sigma}}+\int D \underline{z}^{-} f^{-}\left(z_{5}\right) \frac{\partial g^{-}}{\partial \hat{m}_{\sigma}} .
$$

where the internal derivative will be discussed later.

At this point let us notice that we have 24 saddle point equations arising from the optimisation with respect to the conjugate order parameters, see equations (107)-(130), but above we provided 13 expressions for $D_{i j}, 3$ expressions for $F_{i j}$, the expression for $G^{1, m}$, appearing in 4 equations, and the expression for $M^{1, m}$, describing in total 21 of those saddle point equations. In other words we still need to describe the three equations, namely eq. (110), eq. (113) and eq. (116) and we are going to do it in the following lines, discussing the ansatz mentioned above. First of all, as long as $T>T_{K}$, we can safely set $\hat{Q}_{s s}=Q_{s s}=0$. Moreover, we assume $Q_{\hat{h} s}=Q_{s s \sigma}=0$, so that equations (79), (84), (85) and (86) lead to 
$\hat{Q}_{s s \sigma}=0$ and $\hat{Q}_{\hat{h} s}=\hat{Q}_{\hat{\eta} s}=\hat{Q}_{\hat{\mu} s}=0$. These simplifications allow to write the matrix $U^{ \pm}$, defined in eq. (69), in the following way

$$
U^{ \pm}=\lim _{\hat{Q}_{s s} \rightarrow 0}\left(\begin{array}{ccccc} 
& & & 0 \\
& U_{4}^{ \pm} & & 0 \\
& & & 0 \\
0 & 0 & 0 & 0 & \hat{Q}_{s s}
\end{array}\right), \quad U_{4}^{ \pm}=\left(\begin{array}{cccc}
2 \hat{C}_{\hat{h} \hat{h}} & \hat{C}_{\hat{h} \hat{\eta}} & \hat{C}_{\hat{h} \hat{\mu}} & \pm \hat{C}_{\hat{\hat{h}} s \sigma} \\
\hat{C}_{\hat{h} \hat{\eta}} & \hat{Q}_{\hat{\eta} \hat{\eta}} & \hat{Q}_{\hat{\eta} \hat{\mu}} & \pm \hat{Q}_{\hat{\eta} s \sigma} \\
\hat{C}_{\hat{h} \hat{\mu}} & \hat{Q}_{\hat{\mu} \hat{\mu}} & \hat{Q}_{\hat{\mu} \hat{\mu}} & \pm \hat{Q}_{\hat{\mu} s \sigma} \\
\pm \hat{C}_{\hat{h} s \sigma} & \pm \hat{Q}_{\hat{\eta} s \sigma} & \pm \hat{Q}_{\hat{\mu} s \sigma} & \hat{Q}_{\sigma \sigma}
\end{array}\right)
$$

While checking the self consistency of $Q_{s s}=\hat{Q}_{s s}=0$ is very easy, it is slightly more involved to prove that $Q_{s s \sigma}=Q_{\hat{h} s}=0$ is a self-consistent solutions as well. Let us first consider the condition $Q_{s s \sigma}=0$ and notice that all we need to do is to show the equality $D_{54}^{1, m}=M^{1, m}$, as follows from eq. (108) and (130). $M^{1, m}$, given in eq. (147), contains an inner derivative that we did not discuss before. In fact, given the symmetric role of $\hat{m}_{\sigma}$ and $z_{4}$ in $g^{ \pm}$, see eq. (70), we have

$$
\frac{\partial g^{ \pm}\left(\hat{m}_{\sigma},\{\Delta\}\right)}{\partial \hat{m}_{\sigma}}=\frac{\partial g^{ \pm}\left(\hat{m}_{\sigma},\{\Delta\}\right)}{\partial z_{4}} .
$$

Integrating by parts and differentiating the Gaussian measure, we get

$$
\begin{array}{r}
M^{1, m}=-1+\int D \underline{z}^{+}\left[\sum_{k}\left[U^{+}\right]_{4 k}^{-1} z_{k}\right] f^{+}\left(z_{5}\right) g^{+}\left(\hat{m}_{\sigma},\{\Delta\}\right)+ \\
\int D \underline{z}^{-}\left[\sum_{k}\left[U^{-}\right]_{4 k}^{-1} z_{k}\right] f^{-}\left(z_{5}\right) g^{-}\left(\hat{m}_{\sigma},\{\Delta\}\right) .
\end{array}
$$

Thanks to eq. (148) we can isolate the measure on $z_{5}$ in $D \underline{z}^{ \pm}$,

$$
D \underline{z}^{ \pm} \equiv D \underline{z}_{4}^{ \pm} D z_{5}, \quad D z_{5}=\sqrt{\frac{\hat{Q}_{s s}^{-1}}{2 \pi}} \exp \left[-\frac{1}{2} z_{5}^{2} \hat{Q}_{s s}^{-1}\right] d z_{5}
$$

and, given the definition in eq. (132), it is easy to prove that

$$
\lim _{\hat{Q}_{s s} \rightarrow 0} \int D z_{5} f^{ \pm}\left(z_{5}\right)=\frac{1}{2} \text {. }
$$

Using this equality in eq. (151) we obtain

$$
\begin{aligned}
M^{1, m}=-1+ & \frac{1}{2} \int D \underline{z}_{4}^{+}\left[\sum_{k}\left[U^{+}\right]_{4 k}^{-1} z_{k}\right] g^{+}\left(\hat{m}_{\sigma},\{\Delta\}\right)+ \\
& \frac{1}{2} \int D \underline{z}_{4}^{-}\left[\sum_{k}\left[U^{-}\right]_{4 k}^{-1} z_{k}\right] g^{-}\left(\hat{m}_{\sigma},\{\Delta\}\right)
\end{aligned}
$$

and now we need to show that $D_{54}^{1, m}$ has the very same expression under the ansatz that we are considering. This can be done by noticing that the inverse of the matrix $U^{ \pm}$is block 
diagonal as well

$$
\left[U^{ \pm}\right]^{-1}=\lim _{\hat{Q}_{s s} \rightarrow 0}\left(\begin{array}{ccccc} 
& & & 0 \\
{\left[U_{4}^{ \pm}\right]^{-1}} & & 0 \\
& & & 0 \\
0 & 0 & 0 & 0 & \hat{Q}_{s s}^{-1}
\end{array}\right)
$$

and thus, since $\left[U^{ \pm}\right]_{54}^{-1}=0$, eq. (137) leads to

$$
T_{54}^{ \pm}(\underline{z})=\lim _{\hat{Q}_{s s} \rightarrow 0} \sum_{p}\left[U^{ \pm}\right]_{4 p}^{-1} z_{p} \hat{Q}_{s s}^{-1} z_{5}
$$

$D_{54}^{1, m}$ is given by eq. (136) and, decoupling $D z_{5}$ and $D \underline{z}_{4}^{ \pm}$as in eq. (152), we can write its two contributions as

$$
\begin{aligned}
\int D \underline{z}^{ \pm} T_{54}^{ \pm}(\underline{z}) g^{ \pm}\left(\hat{m}_{\sigma},\{\Delta\}\right) f^{ \pm}\left(z_{5}\right) & =\int D \underline{z}_{4}^{ \pm} D z_{5} \sum_{p}\left[U^{ \pm}\right]_{4 p}^{-1} z_{p} \hat{Q}_{s s}^{-1} z_{5} g^{ \pm}\left(\hat{m}_{\sigma},\{\Delta\}\right) f^{ \pm}\left(z_{5}\right) \\
\int D \underline{z}^{ \pm} T_{54}^{ \pm}(\underline{z}) z_{4} f^{ \pm}\left(z_{5}\right) & =\int D \underline{z}_{4}^{ \pm} D z_{5} \sum_{p}\left[U^{ \pm}\right]_{4 p}^{-1} z_{p} \hat{Q}_{s s}^{-1} z_{5} z_{4} f^{ \pm}\left(z_{5}\right) .
\end{aligned}
$$

We can integrate over $z_{5}$ in both the right hand sides of the equations

$$
\lim _{\hat{Q}_{s s} \rightarrow 0} \int D z_{5} \hat{Q}_{s s}^{-1} z_{5} f^{ \pm}\left(z_{5}\right)= \pm \frac{1}{2}
$$

because $g^{ \pm}\left(\hat{m}_{\sigma},\{\Delta\}\right)$ does not depend on $z_{5}$ and thus we obtain

$$
\begin{aligned}
D_{54}^{1, m}= & -\frac{1}{2} \int D \underline{z}_{4}^{+} \sum_{p}\left[U^{+}\right]_{4 p}^{-1} z_{p} z_{4}-\frac{1}{2} \int D \underline{z}_{4}^{-} \sum_{p}\left[U^{-}\right]_{4 p}^{-1} z_{p} z_{4}+ \\
& +\frac{1}{2} \int D \underline{z}_{4}^{+} \sum_{p}\left[U^{+}\right]_{4 p}^{-1} z_{p} g^{+}\left(\hat{m}_{\sigma},\{\Delta\}\right)+\frac{1}{2} \int D \underline{z}_{4}^{-} \sum_{p}\left[U^{-}\right]_{4 p}^{-1} z_{p} g^{-}\left(\hat{m}_{\sigma},\{\Delta\}\right)(16
\end{aligned}
$$

Finally, integrating over the four dimensional measure the product $z_{p} z_{4}$, we obtain

$$
\begin{aligned}
D_{54}^{1, m}=-1+ & \frac{1}{2} \int D \underline{z}_{4}^{+}\left[\sum_{k}\left[U^{+}\right]_{4 k}^{-1} z_{k}\right] g^{+}\left(\hat{m}_{\sigma},\{\Delta\}\right)+ \\
& \frac{1}{2} \int D \underline{z}_{4}^{-}\left[\sum_{k}\left[U^{-}\right]_{4 k}^{-1} z_{k}\right] g^{-}\left(\hat{m}_{\sigma},\{\Delta\}\right)
\end{aligned}
$$

and so we recognise that $D_{54}^{1, m}=M^{1, m}$, see eq. (154). This strategy can be used also to prove that the zero order terms in the equations leading to $C_{\hat{h} s}, Q_{\hat{h} s}$ and $C_{\hat{h} \hat{h}}$ is zero, i.e. to prove equations (110), (113) and (116), where the second one is needed to check the self-consistency of the considered ansatz. 
The manipulations discussed above lead to a simplified system, that contains only 9 equations, to be compared with the $23+24=47$ equations we had before, that we will describe below. In fact, plugging equations (66), (67) and (68) in eq. (65), and using this equation in eq. (52), we end up with an expression of $S_{\beta}(r)$ that can be written in terms of the original order parameters (i.e. without the conjugate ones) thanks to the equations (78)-(100). In the present ansatz this expression is

$$
\begin{aligned}
S_{\beta}(r)= & \frac{3}{2} Q_{\hat{\mu} s \sigma}^{2}-\frac{3}{2} Q_{\hat{\eta} s \sigma}^{2}+6 Q_{\hat{\mu} s \sigma} Q_{\sigma \sigma} G^{1, m}+3\left[G^{1, m}\right]^{2}\left(Q_{\sigma \sigma}-1\right)+\frac{3}{2} Q_{\sigma \sigma} Q_{\hat{\mu} \hat{\mu}}+ \\
& +\frac{3}{2} Q_{\hat{\eta} \hat{\eta}} Q_{\sigma \sigma}^{2}+4 Q_{\hat{\eta} \hat{\mu}} Q_{\sigma \sigma} m_{\sigma}-\frac{3}{2} Q_{\hat{\eta} \hat{\eta}} Q_{\sigma \sigma}-\frac{3}{2} Q_{\hat{\mu} \hat{\mu}}-3 Q_{\hat{\eta} \hat{\mu}} m_{\sigma}-3 G^{1, m} Q_{\hat{\mu} s \sigma}+ \\
& +3 Q_{\hat{\eta} s \sigma}^{2} Q_{\sigma \sigma}+3 G^{1, m} m_{\sigma} Q_{\hat{\mu} s \sigma}+3 Q_{\hat{\eta} s \sigma} Q_{\hat{\mu} s \sigma} m_{\sigma}-6\left[G^{1, m}\right]^{2}\left(1+m_{\sigma}\right)+ \\
& -3\left[G^{1, m}\right] m_{\sigma} C_{\hat{h} s \sigma}-3 G^{1, m} C_{\hat{h} s \sigma}-\hat{m}_{\sigma} m_{\sigma}+\partial_{m} \Phi
\end{aligned}
$$

where the last term is given by

$$
\partial_{m} \Phi=-\hat{m}_{\sigma}+\frac{1}{2} \int D z_{4}^{+} g^{+}\left(\hat{m}_{\sigma},\{\Delta\}\right)+\frac{1}{2} \int D z_{4}^{-} g^{-}\left(\hat{m}_{\sigma},\{\Delta\}\right) .
$$

As mentioned above, $m_{\sigma}$ is fixed to be $2 r-1$, so that it gives the size of the SSC. The four dimensional measure $D \underline{z}_{4}^{ \pm}$can also be partially expressed in terms of the original order parameters and reads

$$
U_{4}^{ \pm}=\left(\begin{array}{cccc}
\frac{3}{2} & \frac{3}{2} m_{\sigma}^{2} & 3 m_{\sigma} & \pm \hat{C}_{\hat{h} s \sigma} \\
\frac{3}{2} m_{\sigma}^{2} & \frac{3}{2} Q_{\sigma \sigma}^{2} & 3 Q_{\sigma \sigma} m_{\sigma} & \pm \hat{Q}_{\hat{\eta} s \sigma} \\
3 m_{\sigma} & 3 Q_{\sigma \sigma} m_{\sigma} & 3\left(Q_{\sigma \sigma}+m_{\sigma}^{2}\right) & \pm \hat{Q}_{\hat{\mu} s \sigma} \\
\pm \hat{C}_{\hat{h} s \sigma} & \pm \hat{Q}_{\hat{\eta} s \sigma} & \pm \hat{Q}_{\hat{\mu} s \sigma} & \hat{Q}_{\sigma \sigma}
\end{array}\right)
$$

The list of 9 order parameters that we need to determine in order to compute the entropy of SSC at size $r$ is

$$
m_{\sigma}, \quad Q_{\sigma \sigma}, \quad G^{1, m}, \quad C_{\hat{h} s \sigma}, \quad Q_{\hat{\eta} s \sigma}, \quad Q_{\hat{\mu} s \sigma}, \quad Q_{\hat{\eta} \hat{\eta}}, \quad Q_{\hat{\mu} \hat{\mu}}, \quad Q_{\hat{\eta} \hat{\mu}},
$$

as can be seen in eq. (162). Now we are going to show how to compute these order parameters self-consistently. First of all, let us notice that once this list is known, using equations (80), (96), (99) and (100), we obtain

$$
\begin{aligned}
\hat{Q}_{\sigma \sigma} & =3\left(Q_{\hat{\eta} \hat{\eta}} Q_{\sigma \sigma}+Q_{\hat{\eta} s \sigma}^{2}+Q_{\hat{\mu} \hat{\mu}}+\left[G^{1, m}\right]^{2}\right)+6\left(Q_{\hat{\eta} \hat{\mu}} m_{\sigma}+G^{1, m} Q_{\hat{\mu} s \sigma}\right), \\
\hat{C}_{\hat{h} s \sigma} & =3 G^{1, m}\left(1+m_{\sigma}\right), \\
\hat{Q}_{\hat{\eta} s \sigma} & =3\left(Q_{\hat{\eta} s \sigma} Q_{\sigma \sigma}+Q_{\hat{\mu} s \sigma} m_{\sigma}\right), \\
\hat{Q}_{\hat{\mu} s \sigma} & =3\left(Q_{\hat{\mu} s \sigma}+Q_{\hat{\eta} s \sigma} m_{\sigma}+G^{1, m}\left(m_{\sigma}+Q_{\sigma \sigma}\right)\right),
\end{aligned}
$$


and so the matrix $U_{4}^{ \pm}$in eq. (164) is fully specified. Moreover, $m_{\sigma}$ and $Q_{\sigma \sigma}$ completely specify $V$ as well. In fact, using eq. (71) and eq. (72), thanks to the equations (88), (89), (90), (93), (94) and (95), we obtain

$$
V=\left(\begin{array}{cc}
\frac{3}{2}\left(1-Q_{\sigma \sigma}^{2}\right) & 3 m_{\sigma}\left(1-Q_{\sigma \sigma}\right) \\
3 m_{\sigma}\left(1-Q_{\sigma \sigma}\right) & 3\left(1-Q_{\sigma \sigma}\right)
\end{array}\right)
$$

so that $D$ is well defined as well, see eq. (75). Using eq. (81), (82), (83), (97) and (98), the 9 parameters above determine also the following order parameters

$$
\begin{aligned}
\hat{C}_{\hat{h} s} & =\frac{3}{2} \beta \\
\hat{C}_{\hat{\eta} s} & =\frac{3}{2} \beta m_{\sigma}^{2}+3 C_{\hat{h} s \sigma} m_{\sigma}-3 Q_{\hat{\mu} s \sigma} Q_{\sigma \sigma}+3 G^{1, m} \\
\hat{C}_{\hat{\mu} s} & =\frac{3}{2} \beta m_{\sigma}-3 Q_{\hat{\mu} s \sigma} m_{\sigma}+3 G^{1, m} m_{\sigma}-3 G^{1, m} Q_{\sigma \sigma}+3 G^{1, m}+C_{\hat{h} s \sigma} \\
\hat{C}_{\hat{\eta} s \sigma} & =3 G^{1, m}\left(1+m_{\sigma}\right) \\
\hat{C}_{\hat{\mu} s \sigma} & =6 G^{1, m}\left(1+m_{\sigma}\right) .
\end{aligned}
$$

Remembering that $\hat{Q}_{\hat{h} s}=\hat{Q}_{\hat{\eta} s}=\hat{Q}_{\hat{\mu} s}=0$, we can compute $c^{ \pm}$, see eq. $(77)$, and $x_{ \pm}$, see eq. (131). $L_{ \pm}$can be defined as well and so we have everything we need to compute $g^{ \pm}\left(\hat{m}_{\sigma},\{\Delta\}\right)$. All we need to do at this point is to solve self-consistently the equations for the 9 order parameters specified above. For the sake of readability, we report all these equations below:

$$
\begin{aligned}
m_{\sigma}=-1+ & \frac{1}{2} \int D \underline{z}_{4}^{+}\left[\sum_{k}\left[U^{+}\right]_{4 k}^{-1} z_{k}\right] g^{+}\left(\hat{m}_{\sigma},\{\Delta\}\right)+ \\
& \frac{1}{2} \int D \underline{z}_{4}^{-}\left[\sum_{k}\left[U^{-}\right]_{4 k}^{-1} z_{k}\right] g^{-}\left(\hat{m}_{\sigma},\{\Delta\}\right), \\
Q_{\sigma \sigma}= & 1-\frac{1}{2} \int D \underline{z}_{4}^{+} T_{4,4}^{4,+}(\underline{z}) g^{+}\left(\hat{m}_{\sigma},\{\Delta\}\right)-\int D \underline{z}_{4}^{-} T_{4,4}^{4,-}(\underline{z}) g^{-}\left(\hat{m}_{\sigma},\{\Delta\}\right), \\
G^{1, m}= & \frac{1}{2} \int D \underline{z}_{4}^{+} \frac{e^{2\left(\hat{m}_{\sigma}+z_{4}\right)}}{e^{g_{+}}} i_{+}(\underline{z})\left(z_{1}+\hat{C}_{\hat{h}_{s}}\right)-\frac{1}{2} \int D \underline{z}_{4}^{-} \frac{e^{2\left(\hat{m}_{\sigma}+z_{4}\right)}}{e^{g_{-}}} i_{-}(\underline{z})\left(z_{1}-\hat{C}_{\hat{h}_{s}}\right),
\end{aligned}
$$




$$
\begin{aligned}
& C_{\hat{h} s \sigma}=\frac{1}{2} \int D \underline{z}_{4}^{+} T_{4,1}^{4,+}(\underline{z}) g^{+}\left(\hat{m}_{\sigma},\{\Delta\}\right)-\frac{1}{2} \int D \underline{z}_{4}^{-} T_{4,1}^{4,-}(\underline{z}) g^{-}\left(\hat{m}_{\sigma},\{\Delta\}\right), \\
& Q_{\hat{\eta} s \sigma}=-\frac{1}{2} \int D \underline{z}_{4}^{+} T_{4,2}^{4,+}(\underline{z}) g^{+}\left(\hat{m}_{\sigma},\{\Delta\}\right)+\frac{1}{2} \int D \underline{z}_{4}^{-} T_{4,2}^{4,-}(\underline{z}) g^{-}\left(\hat{m}_{\sigma},\{\Delta\}\right) \\
& +\frac{1}{2} \int D \underline{z}_{4}^{+} \frac{e^{2\left(z_{4}+\hat{m}_{\sigma}\right)}}{e^{g_{+}}} \frac{e^{-x_{+}^{2}}}{\sqrt{\pi}} \frac{\operatorname{sgn}\left(a_{+}\right)}{\sqrt{2 D}}-\frac{1}{2} \int D \underline{z}_{4}^{-} \frac{e^{2\left(z_{4}+\hat{m}_{\sigma}\right)}}{e^{g_{-}}} \frac{e^{-x_{-}^{2}}}{\sqrt{\pi}} \frac{\operatorname{sgn}\left(a_{-}\right)}{\sqrt{2 D}}, \\
& Q_{\hat{\mu} s \sigma}=-\frac{1}{2} \int D \underline{z}_{4}^{+} T_{4,3}^{4,+}(\underline{z}) g^{+}\left(\hat{m}_{\sigma},\{\Delta\}\right)+\frac{1}{2} \int D \underline{z}_{4}^{-} T_{4,3}^{4,-}(\underline{z}) g^{-}\left(\hat{m}_{\sigma},\{\Delta\}\right) \\
& +\frac{1}{2} \int D \underline{z}_{4}^{+} \frac{e^{2\left(z_{4}+\hat{m}_{\sigma}\right)}}{e^{g_{+}}} \frac{e^{-x_{+}^{2}}}{\sqrt{\pi}} \frac{\operatorname{sgn}\left(a_{+}\right)}{\sqrt{2 D}}-\frac{1}{2} \int D \underline{z}_{4}^{-} \frac{e^{2\left(z_{4}+\hat{m}_{\sigma}\right)}}{e^{g_{-}}} \frac{e^{-x_{-}^{2}}}{\sqrt{\pi}} \frac{\operatorname{sgn}\left(a_{-}\right)}{\sqrt{2 D}}, \\
& Q_{\hat{\eta} \hat{\eta}}=-\int D \underline{z}_{4}^{+} T_{22}^{4,+}(\underline{z}) g^{+}\left(\hat{m}_{\sigma},\{\Delta\}\right)-\int D \underline{z}_{4}^{-} T_{22}^{4,-}(\underline{z}) g^{-}\left(\hat{m}_{\sigma},\{\Delta\}\right)+ \\
& -\int D \underline{z}_{4}^{+} \frac{e^{2\left(z_{4}+\hat{m}_{\sigma}\right)}}{e^{g_{+}}} \frac{e^{-x_{+}^{2}}}{\sqrt{\pi}} \frac{x_{+}}{2 D}-\int D \underline{z}_{4}^{-} \frac{e^{2\left(z_{4}+\hat{m}_{\sigma}\right)}}{e^{g_{-}}} \frac{e^{-x_{+}^{2}}}{\sqrt{\pi}} \frac{x_{-}}{2 D}, \\
& Q_{\hat{\mu} \hat{\mu}}=-\int D \underline{z}_{4}^{+} T_{33}^{4,+}(\underline{z}) g^{+}\left(\hat{m}_{\sigma},\{\Delta\}\right)-\int D \underline{z}_{4}^{-} T_{33}^{4,-}(\underline{z}) g^{-}\left(\hat{m}_{\sigma},\{\Delta\}\right)+ \\
& -\int D \underline{z}_{4}^{+} \frac{e^{2\left(z_{4}+\hat{m}_{\sigma}\right)}}{e^{g_{+}}} \frac{e^{-x_{+}^{2}}}{\sqrt{\pi}} \frac{x_{+}}{2 D}-\int D \underline{z}_{4}^{-} \frac{e^{2\left(z_{4}+\hat{m}_{\sigma}\right)}}{e^{g_{-}}} \frac{e^{-x_{+}^{2}}}{\sqrt{\pi}} \frac{x_{-}}{2 D}, \\
& Q_{\hat{\eta} \hat{\mu}}=-\frac{1}{2} \int D \underline{z}_{4}^{+} T_{23}^{4,+}(\underline{z}) g^{+}\left(\hat{m}_{\sigma},\{\Delta\}\right)-\frac{1}{2} \int D \underline{z}_{4}^{-} T_{23}^{4,-}(\underline{z}) g^{-}\left(\hat{m}_{\sigma},\{\Delta\}\right)+ \\
& -\int D \underline{z}_{4}^{+} \frac{e^{2\left(z_{4}+\hat{m}_{\sigma}\right)}}{e^{g_{+}}} \frac{e^{-x_{+}^{2}}}{\sqrt{\pi}} \frac{x_{+}}{2 D}-\int D \underline{z}_{4}^{-} \frac{e^{2\left(z_{4}+\hat{m}_{\sigma}\right)}}{e^{g_{-}}} \frac{e^{-x_{+}^{2}}}{\sqrt{\pi}} \frac{x_{-}}{2 D},
\end{aligned}
$$

where, consistently with the notation adopted in eq. (137), we defined

$$
T_{i j}^{4, \pm}(\underline{z})=\left\{\begin{array}{c}
\sum_{l p}\left[U_{4}^{ \pm}\right]_{l i}^{-1}\left[U_{4}^{ \pm}\right]_{p j}^{-1} z_{l} z_{p}-\left[U_{4}^{ \pm}\right]_{i j}^{-1} \quad i \neq j \\
\frac{1}{2} \sum_{l p}\left[U_{4}^{ \pm}\right]_{l i}^{-1}\left[U_{4}^{ \pm}\right]_{p i}^{-1} z_{l} z_{p}-\frac{1}{2}\left[U_{4}^{ \pm}\right]_{i i}^{-1} i=j
\end{array}, \quad \underline{z}=\left\{z_{1}, z_{2}, z_{3}, z_{4}\right\} .\right.
$$

This is a complicated system of equations that can be solved numerically by iteration. For each given value of $\hat{m}_{\sigma}$ and $\beta$, we start with some trial values of the order parameters listed in eq. (165). We compute all the quantities discussed above in order to perform the integrals in equations (176)-(184) and we end up with a new list of order parameters. Integrals over the Gaussian measures are computed using a MonteCarlo sampling method. The number of random 4-dimensional vectors that we need to sample in order to get reliable results is $O\left(10^{8}\right)$. We iterate this process until convergence by computing the difference 
between the old and the new order parameters starting from different initial conditions. The first equation provides the $r$ which corresponds to the initial choice of $\hat{m}_{\sigma}$ and $\beta$. The corresponding entropy is then provided from eq. (162). Usually, $O(200)$ iteration steps are required to get a good solution. We never find multiple solutions starting from different initial conditions but the quality of the solution is found to depend on $r$. In particular, when $r$ is very close to 1 , the error on the final result is larger because it becomes more and more difficult to invert the matrices $U$ and $V$. By the way, as the result presented in Fig. (1) of the Main Text show, this is not a practical problems, since as long as solutions can be found the profile of the entropy $S_{\beta}(r)$ is very smooth.

Finally, we would like to discuss how to compute the distribution of local fields in SSC recycling all the effort made up to now. To this aim, let us introduce

$$
N S_{\beta}^{\gamma}(r)=\mathbb{E}_{J} \mathbb{E}_{\underline{s}} \log \sum_{\underline{\sigma}} \mathbb{I}_{\underline{\sigma}}^{\gamma}(\underline{s}) \delta\left(r N-\sum_{i=1}^{N} \frac{1+\sigma_{i}}{2}\right)
$$

where $\mathbb{I}_{\underline{\sigma}}^{\gamma}(\underline{s})$, given eq. (5), is defined by

$$
\mathbb{I}_{\underline{\sigma}}^{\gamma}(\underline{s})=\prod_{i=1}^{N}\left\{\frac{1-\sigma_{i}}{2}+\frac{1+\sigma_{i}}{2} \theta\left[u_{i}^{2}-\left(v_{i}+w_{i}\right)^{2}-\epsilon\right] e^{\gamma \delta\left(h_{i}-\lambda\right)}\right\} .
$$

This quantity has two important properties. The trivial one is that $\lim _{\gamma \rightarrow 0} S_{\beta}^{\gamma}(r)=S_{\beta}(r)$. The less trivial one is that the derivative of this function leads to eq. (8). In fact, let us compute

$$
N \lim _{\gamma \rightarrow 0} \partial_{\gamma} S_{\beta}^{\gamma}(r)=\mathbb{E}_{J} \mathbb{E}_{\underline{s}}\left[\sum_{\underline{\sigma}} \mathbb{I}_{\underline{\sigma}}^{r}(\underline{s})\right]^{-1} \sum_{\underline{\sigma}, i} \mathbb{I}_{\underline{\sigma}}^{(i), r}(\underline{s})\left(\frac{1+\sigma_{i}}{2}\right) \theta\left[u_{i}^{2}-\left(v_{i}+w_{i}\right)^{2}-\epsilon\right] \delta\left(h_{i}-\lambda\right)
$$

where $\mathbb{I}_{\underline{\sigma}}^{r}(\underline{s})$ has been defined in eq. (10) and $\mathbb{I}_{\underline{\sigma}}^{(i), r}(\underline{s})$ is given by

$$
\mathbb{I}_{\underline{\sigma}}^{(i), r}(\underline{s})=\mathbb{I}_{\underline{\sigma}}^{(i)}(\underline{s}) \delta\left(r N-\sum_{i=1}^{N} \frac{1+\sigma_{i}}{2}\right)
$$

where

$$
\mathbb{I}_{\underline{\sigma}}^{(i)}(\underline{s})=\prod_{j \neq i}\left[\frac{1-\sigma_{j}}{2}+\frac{1+\sigma_{j}}{2} \theta\left[u_{j}^{2}-\left(v_{j}+w_{j}\right)^{2}-\epsilon\right]\right] .
$$

In order to recognize that the right hand side of eq. (188) is strictly related to eq. (8), we only need to show that

$$
\mathbb{I}_{\underline{\sigma}}^{(i), r}(\underline{s})\left(\frac{1+\sigma_{i}}{2}\right) \theta\left[u_{i}^{2}-\left(v_{i}+w_{i}\right)^{2}-\epsilon\right]=\mathbb{I}_{\underline{\sigma}}^{r}(\underline{s})\left(\frac{1+\sigma_{i}}{2}\right) .
$$


This equality can be verified by the following considerations. First of all let us notice that if $\sigma_{1}=-1$, eq. (191) is trivially satisfied. Moreover, the indicator function in $\mathbb{I}_{\underline{\sigma}}^{r}(\underline{s})$, given in eq. (5), is one if and only if the cluster specified by $\underline{\sigma}$ (i.e. those spins where $\sigma_{i}=1$ ) is self-sustained and the same condition holds for $\mathbb{I}_{\underline{\sigma}}^{(i)}(\underline{s})$ that, as seen in the definition in eq. (189), is one if and only if the cluster specified by $\underline{\sigma} \backslash \sigma_{i}$ is self-sustained. Thus, even when $\sigma_{1}=1$, we see that $\mathbb{I}_{\underline{\sigma}}^{(i)}(\underline{s})$ in the left hand side lacks a condition on the spin $s_{i}$ that is explicitly enforced by the Heaviside function. Thus, eq. (188) leads to

$$
r^{-1} \lim _{\gamma \rightarrow 0} \partial_{\gamma} S_{\beta}^{\gamma}(r)=P_{r}(h=\lambda) .
$$

Numerically, we worked with a small value of $\gamma=0.1$ and we solved the two sets of saddle point equations with $\gamma=0$ and $\gamma \neq 0$. In the second case, all we need to do is replacing eq. (5) with eq. (187) and repeat the derivation illustrated above. The only difference arises when computing the integrals over $D \underline{x}$ in eq. (73):

$$
L_{ \pm}=\int D \underline{x} \theta_{\underline{x}, \underline{z}}^{ \pm}(\{\Delta\}) \longmapsto L_{ \pm}^{\gamma}=\int D \underline{x} \theta_{\underline{x}, \underline{z}}^{ \pm}(\{\Delta\}) e^{\gamma \delta\left(h_{i}-\lambda\right)}
$$

where $h_{i}$ is actually independent of $\underline{x}$, and it is given by eq. (60). Since we are only interested in the limit $\gamma \sim 0$, we can replace

$$
e^{\gamma \delta\left(h_{i}-\lambda\right)} \sim 1+\gamma \delta\left(h_{i}-\lambda\right)=1+\gamma \delta\left(z_{1} \pm \Delta_{\hat{h} s}-\lambda\right)
$$

and, in the numerical iteration process, we approximated the delta function by a Gaussian

function with a small variance. Subtracting $S_{\beta}^{\gamma=0}(r)$ from $S_{\beta}^{\gamma=0.1}(r)$, we computed the points in the inset of Fig. (4). Continuous lines are then obtained by fitting Gaussians to these points.

\section{Exhaustive Calculation of SSC Entropy in Small Systems}

To verify our analytical results, we calculate the empirical entropy of SSC in small $p$-spin systems. As we have discussed in the main paper, the entropy $S_{\beta}(r)$ of SSC is given by Eqs. $(4)-(6)$

$$
\mathcal{S}_{\beta}(r)=\mathbb{E}_{J} \mathbb{E}_{\underline{s}}\left[\mathcal{S}\left(r \mid \underline{s},\left\{J_{i j k}\right\}\right)\right]
$$

where

$$
\mathcal{S}\left(r \mid \underline{s},\left\{J_{i j k}\right\}\right)=\frac{1}{N} \log \sum_{\underline{\sigma}} \mathbb{I}_{\underline{\sigma}}(\underline{s}) \delta\left(r N-\sum_{i=1}^{N} \frac{1+\sigma_{i}}{2}\right)
$$


which leads to

$$
\mathcal{S}_{\beta}(r)=\mathbb{E}_{J} \mathbb{E}_{\underline{s}}\left[\frac{1}{N} \log \sum_{\underline{\sigma}} \mathbb{I}_{\underline{\sigma}}(\underline{s}) \delta\left(r N-\sum_{i=1}^{N} \frac{1+\sigma_{i}}{2}\right)\right] .
$$

However, in small systems, SSC of specific size may be absent in some spin configurations due to finite-size effect. This implies that for these particular spin configurations, $\mathbb{I}_{\underline{\sigma}}(\underline{s})=0$ for some $r$ such that the logarithm and hence $\mathcal{S}\left(r \mid \underline{s},\left\{J_{i j k}\right\}\right)$ become negative infinity for some $r$. In order to compute the entropy of SSC, one has to average $\mathcal{S}\left(r \mid \underline{s},\left\{J_{i j k}\right\}\right)$ over all spin configurations subject to Boltzmann weights. If there exists at least one spin configuration where SSC of size $r$ are absent, and if the same happen for all $r$, then $\mathbb{E}_{\underline{s}}\left[\mathcal{S}\left(r \mid \underline{s},\left\{J_{i j k}\right\}\right)\right]=0$ for all $r$. In other words, the entropy of SSC becomes undefined even there are SSC for a majority of spin configurations.

To cope with this problem in the exhaustive calculation on small systems, we modify the definition of the entropy of SSC as follows:

$$
\mathcal{S}_{\beta}(r)=\mathbb{E}_{J}\left\{\frac{1}{N} \log \mathbb{E}_{\underline{s}}\left[\sum_{\underline{\sigma}} \mathbb{I}_{\underline{\sigma}}(\underline{s}) \delta\left(r N-\sum_{i=1}^{N} \frac{1+\sigma_{i}}{2}\right)\right]\right\} .
$$

In this case, for a particular set of quenched disorders $J$, the number of SSC is averaged over spin configurations before the logarithm is taken for the computation of entropy. This expression is different from Eq. (195), where the entropy of SSC is computed for individual spin configuration and the average over spin configurations is taken afterwards. As a result, the empirical results obtained by Eq. (196) are in principle different from the analytical results obtained by Eq. (195), but the comparison between the two would still serve as a good mean of verification for the analytical results.

Regarding the computational complexity of the calculation, the exact computation of SSC entropy on a system of size $N$ involves an average over all spin configurations and the summation over all $\underline{\sigma}$, resulting in $2^{2 N}$ computations which are computationally infeasible even for $N \approx 20$. We thus sample spin configurations according to Boltzmann weights at temperature $T$ instead of averaging over all spin configurations. For each of the sampled spin configuration, we compute the number of SSC with size $r$ by exhaustive search over all $\underline{\sigma}$. By averaging the number of SSC with size $r$ and taking the logarithm, we obtain the SSC entropy of small systems given by Eq. (196). The results are shown in Fig. 2 of the main paper, which matches qualitatively with the analytical results. 


\section{E. The decomposition of the field in a general $p$-spin model}

According to Eq. (2) of the main text, one can split the contributions to the local field $h_{i}$ of a spin $i$ in a 3 -spin model into the internal field $u_{i}$, the external field $v_{i}$ or mixed field $w_{i}$. For a spin $i$ in a general $p$-spin model, the corresponding fields are defined similarly as

$$
h_{i}=\frac{1}{(p-1) !} \sum_{j_{1}, \ldots, j_{p}} J_{i j_{1}, \ldots, j_{p}} s_{j_{1}}, \ldots, s_{j_{p}}=\frac{1}{(p-1) !}\left(u_{i}+t_{i}\right),
$$

where

$$
\begin{aligned}
u_{i} & =\sum_{j_{1}, \ldots, j_{p} \in C} J_{i j_{1}, \ldots, j_{p}} s_{j_{1}}, \ldots, s_{j_{p}}, \\
t_{i} & =\sum_{\left\{j_{1}, \ldots, j_{p} \mid j_{1} \cup j_{2} \cup \cdots \cup j_{p} \notin C\right\}} J_{i j_{1}, \ldots, j_{p}} s_{j_{1}}, \ldots, s_{j_{p}} .
\end{aligned}
$$

In the second term, at least one of the spins we are summing over does not belong to cluster $C$. In the 3 -spin case, $t_{i}$ corresponds to the sum of $v_{i}$ and $w_{i}$ defined in Eq. (2) of the main text. 